\title{
ASTROMETRY, RADIAL VELOCITY, AND PHOTOMETRY: THE HD 128311 SYSTEM REMIXED WITH DATA FROM HST, HET, AND APT*
}

\author{
Barbara. E. McArthur ${ }^{1}$, G. Fritz. Benedict ${ }^{1}$, Gregory W. Henry ${ }^{2}$, Artie Hatzes ${ }^{3}$, William D. Cochran ${ }^{1}$, \\ Tom E. Harrison ${ }^{4}, 7$, Chris Johns-KrulL ${ }^{5}$, AND Ed Nelan 6 \\ ${ }^{1}$ McDonald Observatory, University of Texas at Austin, Austin, TX 78712, USA; mca@ astro.as.utexas.edu \\ ${ }^{2}$ Center of Excellence in Information Systems, Tennessee State University, 3500 John A. Merritt Blvd., P.O. Box 9501, Nashville, TN 37209, USA \\ ${ }^{3}$ Thüringer Landessternwarte, Sternwarte 5, D-07778 Tautenburg, Germany \\ ${ }^{4}$ Department of Astronomy, New Mexico State, Las Cruces, NM 88003, USA \\ ${ }^{5}$ Department of Physics and Astronomy, Rice University, MS-108, 6100 Main Street, Houston, TX 77005, USA \\ ${ }^{6}$ Space Telescope Science Institute, 3700 San Martin Drive, Baltimore, MD 21218, USA \\ Received 2013 April 4; accepted 2014 September 3; published 2014 October 10
}

\begin{abstract}
We have used high-cadence radial velocity measurements from the Hobby-Eberly Telescope with published velocities from the Lick $3 \mathrm{~m}$ Shane Telescope, combined with astrometric data from the Hubble Space Telescope (HST) Fine Guidance Sensors to refine the orbital parameters of the HD 128311 system, and determine an inclination of $55^{\circ} .95 \pm 14.55$ and true mass of $3.789_{-0.432}^{+0.924} M_{\mathrm{JUP}}$ for HD $128311 \mathrm{c}$. The combined radial velocity data also reveal a short period signal which could indicate a third planet in the system with an $M \sin i$ of $0.133 \pm 0.005 M_{\mathrm{JUP}}$ or stellar phenomena. Photometry from the T12 $0.8 \mathrm{~m}$ automatic photometric telescope at the Fairborn Observatory and HST are used to determine a photometric period close to, but not within the errors of the radial velocity signal. We performed a cross-correlation bisector analysis of the radial velocity data to look for correlations with the photometric period and found none. Dynamical integrations of the proposed system show long-term stability with the new orbital parameters of over 10 million years. Our new orbital elements do not support the claims of HD $128311 \mathrm{~b}$ and $\mathrm{c}$ being in mean motion resonance.
\end{abstract}

Key words: astrometry - parallaxes - planetary systems - planets and satellites: dynamical evolution and stability - stars: individual (HD 128311, Upsilon Andromedae)

Online-only material: color figures, machine-readable tables

\section{INTRODUCTION}

HD 128311 is a variable $\mathrm{K} 3 \mathrm{~V}$ (alternatively K0 V) star of BY Dra-type that is approximately 0.5 Gyr old and $16.57 \mathrm{pc}$ from Earth, younger and less massive than our Sun. It is a probable member of the Ursa Major moving group (Maldonado et al. 2010). The first planet found around HD 128311, with a period of 422 days, was announced in 2003, discovered with highprecision radial velocity (RV) measurements by Butler et al. (2003), who also noted a linear trend in the data (which could also indicate and additional longer period companion). In 2005, a second planet, with a period of 928 days, was announced and a signal found at 5.6 days was attributed to starspots (Vogt et al. 2005).

The early published RV orbital parameters were unstable in dynamic integrations (Vogt et al. 2005; Meschiari et al. 2009). The suggestion was made that mean motion resonance (MMR) was a path to stability, and many studies were performed on the system in MMR (Vogt et al. 2005; Beaugé et al. 2006; Quillen 2006; Sándor \& Kley 2006; Sándor et al. 2007; Michtchenko et al. 2008; Meschiari et al. 2009; Crida et al. 2008;

\footnotetext{
* Based on observations made with the NASA/ESA Hubble Space Telescope, obtained at the Space Telescope Science Institute, which is operated by the Association of Universities for Research in Astronomy, Inc., under NASA contract NAS5-26555. Based on observations obtained with the Hobby-Eberly Telescope, which is a joint project of the University of Texas at Austin, the Pennsylvania State University, Stanford University,

Ludwig-Maximilians-Universität München, and Georg-August-Universität Göttingen, and observations with T12 $0.8 \mathrm{~m}$ automatic photoelectric telescope (APT) at Fairborn Observatory.

7 Guest observer at the Cerro Tololo Inter-American Observatory (CTIO), a division of the National Optical Astronomy Observatory, which is operated by the Association of Universities for Research in Astronomy, Inc., under cooperative agreement with the National Science Foundation.
}

Lee \& Thommes 2009; Voyatzis et al. 2009). Some invoked planet-planet scattering as a pathway to the resonance (Barnes \& Greenberg 2006a, 2006b; Raymond et al. 2008) and some used turbulence to explain the broken apsidal corotation while the system is thought to be in MMR (Rein \& Papaloizou 2009; Lecoanet et al. 2009). In addition to the most common 2:1 MMR suggestion, a retrograde MMR was explored (Gayon \& Bois 2008; Gayon-Markt \& Bois 2009) and, alternatively, a Trojan configuration of planets in highly inclined orbits in 1:1 MMR was proposed (Goździewski \& Konacki 2006; Érdi et al. 2007) which was later suggested to be quasi-satellite trajectories instead of the Trojan configuration (Giuppone et al. 2010).

An important complement to the discovery of extrasolar planets is the discovery of disks around these multiple planet-bearing stars, which can elaborate the possibility of interactions which could produce these detectable excesses. A study searching for disks around exoplanet host stars using the criteria proposed by Mannings \& Barlow (1998) identified an IR excess that may have indicated the presence of dust in a circumstellar disk around HD 128311 (Saffe \& Gómez 2004). Emission was found at $70 \mu \mathrm{m}$ with the Spitzer MIPS instrument, but no evidence for excess is seen out to $30 \mu \mathrm{m}$ (Beichman et al. 2005). Using the Infrared Spectrograph (IRS) on Spitzer, no excess was found at $5-35 \mu \mathrm{m}$ and single grain modeling projected that the disk seen at $70 \mu \mathrm{m}$ must start beyond $15 \mathrm{AU}$ (Beichman et al. 2006), similar to the solar system's Kuiper Belt. Trilling et al. (2008) found a fractional luminosity ( $f_{d}$ at $70 \mu \mathrm{m}$ maximum of $2.7 \%$ and minimum of $1.3 \%$ ). Bryden et al. (2009) regarded this as a relatively weak detection, with no excess at $24 \mu \mathrm{m}$ detected with MIPS. Spitzer observations found no excess at $160 \mu \mathrm{m}$ (Tanner et al. 2009). Lawler et al. (2009) found that HD 128311 was 1 of 3 stars out of 152 FGKM stars that had a $70 \mu \mathrm{m}$ excess 
Table 1

Mass and Radius of HD 128311

\begin{tabular}{lclc}
\hline \hline ID & Value & Unit & Reference \\
\hline$M_{*}$ & 0.84 & $M_{\odot}$ & Vogt et al. 2005 \\
$M_{*}$ & $0.828-0.012+0.008$ & $M_{\odot}$ & Takeda et al. 2007 \\
$R_{*}$ & $0.73 \pm 0.016$ & $R_{\odot}$ & Valenti \& Fischer 2005 \\
$R_{*}$ & $0.78 \pm 0.02$ & $R_{\odot}$ & van Belle \& von Braun 2009 \\
$R_{*}$ & $0.762 \pm 0.013$ & $R_{\odot}$ & Ribas et al. 2003 \\
$R_{*}$ & $0.76 \pm 0.02$ & $R_{\odot}$ & Takeda et al. 2007 \\
\hline
\end{tabular}

without a corresponding excess at $33 \mu \mathrm{m}$. The detection of the cool dust at $70 \mu \mathrm{m}$ in HD 128311, indicates that there are most likely still collisions in this system, coming from the interaction of large planetesimal, small undetected planetary perturbers, or from effects of interactions of the known planets.

HD 128311 is one of the handful of systems (currently six; Moro-Martín et al. 2010) known to contain planets and debris disks, which further enhances its role in the study of formation and evolution of extrasolar planetary systems. Like $\epsilon$ Eridani and HD 69830, HD 128311 also has an extensive debris belt (Trilling et al. 2008) at a radius of >5.1 AU. Moro-Martín et al. (2010) used Spitzer IRS spectra to predict that the location of planetesimals in the system was between 52 and $100 \mathrm{AU}$, while their dynamical modeling indicated two regions of stability for planetesimal lifetimes: interior to $0.3 \mathrm{AU}$ and exterior to $4 \mathrm{AU}$, which is preferred because there is no excess emission at $\lambda<33 \mu \mathrm{m}$.

Because extrasolar planetary systems are expected to produce low-frequency radio emissions, as we detect in our own planetary system, a search was made for these emissions from the planets around HD 128311 (George \& Stevens 2007). No detection was found, but an upper limit of $15.5 \mathrm{mJy}$ for radio emissions was placed on the system.

We have used the Hubble Space Telescope (HST) Fine Guidance Sensor (FGS) 1r to obtain millisecond of arc per observation precision astrometry which we combine with new Hobby-Eberly Telescope (HET) RVs and archival Lick velocity sets. In this paper, we use the combined astrometric and spectroscopic measurements to calculate the actual mass of planet $\mathrm{c}$, show evidence of a third shorter period planet or star phenomenon, and examine the resonance and stability of the system. We use T12 automatic photometric telescope (APT) and $H S T$ photometry to investigate the stellar activity in this system.

\section{STELLAR PROPERTIES}

HD $128311\left(=\mathrm{V}^{*}\right.$ HN Boo $=$ HIP71395 = GJ3860+RX $\mathrm{J} 1436.0+0944)$ is a $V=7.446, \mathrm{~K} 3 \mathrm{~V}$ star with a mass of $0.828 M_{\odot}$ and radius of $0.76 M_{\odot}$ (see Table 1 ). Table 2 summarizes the observed properties of HD 128311, a star with roughly solar metallicity. Observations of $\mathrm{Ca} \mathrm{II} \mathrm{H}$ and $\mathrm{K}$ lines indicate high chromospheric activity for this star (Butler et al. 2003; Vogt et al. 2005). Butler's initial estimates of photospheric velocity variability were between 30 and $100 \mathrm{~m} \mathrm{~s}^{-1}$ (Butler et al. 2003). Later estimates suggested the variability (sometimes referred to as jitter) was $8.9 \mathrm{~m} \mathrm{~s}^{-1}$ (Vogt et al. 2005). The Ca II H and $\mathrm{K}$ lines also showed strong emission reversals in the High Resolution Echelle Spectrometer spectra, which implies a young age of 0.5-1.0 Gyr (Vogt et al. 2005). All age estimates can be seen in Table 3. Though we have chosen specific measurements to use in our calculations we list alternative measurements for ease of comparison, and disclosure of the star properties.
Table 2

Stellar Properties of HD 128311

\begin{tabular}{lccc}
\hline \hline ID & Value & Unit & Reference \\
\hline Spectral type & $\mathrm{K} 3 \mathrm{~V}$ & $\ldots$ & Gray et al. 2003 \\
Spectral type & $\mathrm{K} 0 \mathrm{~V}$ & $\ldots$ & Vogt et al. 2005 \\
$v \sin i$ & 3.65 & $\mathrm{~km} \mathrm{~s}^{-1}$ & Fischer \& Valenti 2005 \\
$A_{V}$ & $0.59 \pm 0.013$ & $\mathrm{mag}$ & van Belle \& von Braun 2009 \\
$B$ & 8.55 & $\mathrm{mag}$ & Bailer-Jones 2011 \\
$V$ & $7.51 \pm 0.05$ & $\mathrm{mag}$ & Bailer-Jones 2011 \\
$R$ & 6.88 & $\mathrm{mag}$ & Zacharias et al. 2004 \\
$I$ & 6.711 & $\mathrm{mag}$ & Droege et al. 2006 \\
$J$ & $5.772 \pm 0.018$ & $\mathrm{mag}$ & Bailer-Jones 2011 \\
$H$ & $5.303 \pm 0.031$ & $\mathrm{mag}$ & Bailer-Jones 2011 \\
$K$ & $5.142 \pm 0.017$ & $\mathrm{mag}$ & Bailer-Jones 2011 \\
$T_{\text {eff }}$ & $4815 \pm 53$ & $\mathrm{~K}$ & Ramírez \& Meléndez 2005 \\
$T_{\text {eff }}$ & 4965 & $\mathrm{~K}$ & Valenti \& Fischer 2005 \\
$T_{\text {eff }}$ & 4763 & $\mathrm{~K}$ & Saffe et al. 2008 \\
$\log g$ & $4.83 \pm 0.1$ & $\mathrm{~cm} \mathrm{~s}^{-2}$ & Valenti \& Fischer 2005 \\
$\log g$ & $4.61-0.02+0.01$ & $\mathrm{~cm} \mathrm{~s}^{-2}$ & Takeda et al. 2007 \\
{$[$ Fe $/ \mathrm{H}]$} & 0.2 & $\mathrm{dex}$ & Valenti \& Fischer 2005 \\
{$[$ Fe $/ \mathrm{H}]$} & 0.1 & $\mathrm{dex}$ & Ramírez \& Meléndez 2005 \\
{$[$ Fe $/ \mathrm{H}]$} & $0.023 \pm 0.081$ & $\mathrm{dex}$ & Gonzalez \& Laws 2007 \\
$X$-ray flux & $10.2 \pm 1.35$ & $10^{-13} \mathrm{erg} \mathrm{cm}^{-2} \mathrm{~s}^{-1}$ & Kashyap et al. 2008 \\
\hline & & &
\end{tabular}

Note. ${ }^{a}$ Castelli et al. (1997) calibration.

Table 3

Age Estimates of HD 128311

\begin{tabular}{lccc}
\hline \hline ID & Value & Unit & Reference \\
\hline Age & $<0.39$ & Gyr & Saffe et al. 2005 \\
Age & $<0.41$ & Gyr & Saffe et al. 2005 \\
Age & $<6.4$ & Gyr & Valenti \& Fischer 2005 \\
Age & $<0.960 \pm 0.04$ & Gyr & Barnes 2007 \\
Age & 0.351 & Gyr & Barnes 2007 \\
Lower age limit & $<0.7$ & Gyr & Valenti \& Fischer 2005 \\
Upper age limit & $<12.7$ & Gyr & Valenti \& Fischer 2005 \\
Upper age limit & $<0.96$ & Gyr & Takeda et al. 2007 \\
\hline
\end{tabular}

Notes.

${ }^{a}$ Chromospheric age using the calibration of Donahue (1993).

${ }^{\mathrm{b}}$ Chromospheric age using the calibration of Rocha-Pinto \& Maciel (1998).

c Isochrone age.

d Gyrochronology used for age.

\section{OBSERVATIONS, DATA REDUCTION, AND MODELING}

\subsection{HET and Lick Radial Velocity Observations}

The High Resolution Spectrograph (HRS; Tull 1998) at the HET at McDonald Observatory was used to make the spectroscopic observations using the iodine absorption cell method (Butler et al. 1996). Our reduction of HET HRS data is given in Bean et al. (2007), which uses the REDUCE package (Piskunov \& Valenti 2002). Our observations include a total of 355 high-resolution spectra which were obtained between 2005 April and 2011 January. Because typically two or more observations were made in less than $1 \mathrm{hr}$ per night, we observed at 161 epochs with the HET HRS.

Our HET RV data set was combined with overlapping velocities from Lick taken from Meschiari et al. (2009) to produce a total data set that spans $13 \mathrm{yr}$. The total RV data set contained 455 observations of HD 128311. Table 4 contains reduced HET velocities for the observed epochs. We removed a few HET RV observations from our data set based upon the analysis discussed in Section 4.4. 


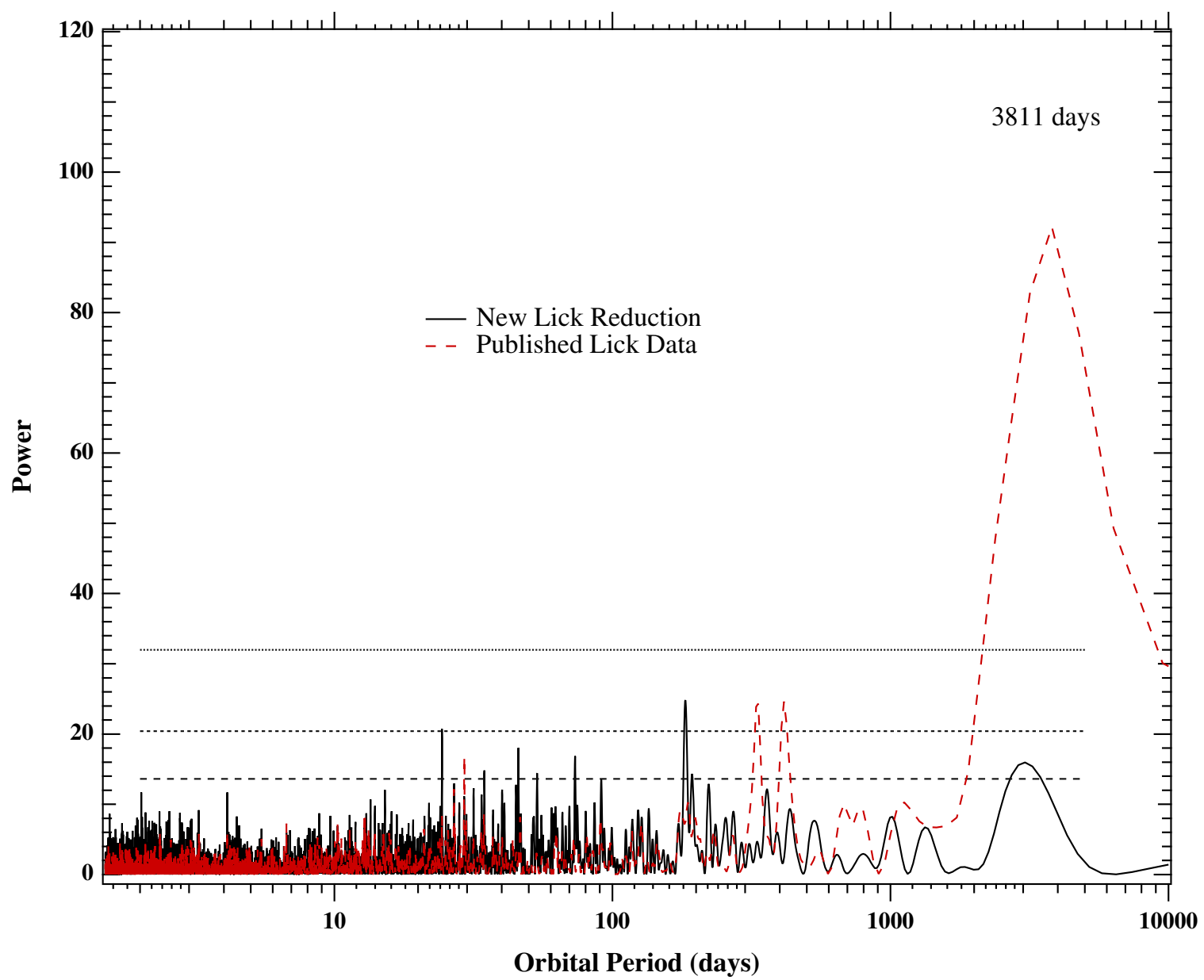

Figure 1. Periodogram of the radial velocities of $v$ Andromedae from two reductions of Lick data: the published (Wright et al. 2009) and the new lick reduction (D. Fisher \& M. Giguere 2009, private communication). Shows the two periodogram of the residuals to a Keplerian model that contains the planet b (period 4.617 days), planet c (241 days), and planet d (1282 days). The significance of the signal at 3811 days (identified as $v$ Andromedae by Curiel et al. 2011) is considerably diminished in the new Lick reduction. The dotted lines show the significance levels of the power in each plot, from the top: 1.0e10, 1.0e5, and 1.0e2.

(A color version of this figure is available in the online journal.)

Table 4

HET Relative Radial Velocities for HD 128311

\begin{tabular}{lcc}
\hline \hline JD- 2450000 & RV $\left(\mathrm{m} \mathrm{s}^{-1}\right)$ & \pm Error $^{\mathrm{a}}$ \\
\hline 3462.965290 & 97.99 & 6.99 \\
3479.731833 & 82.71 & 6.97 \\
3479.899542 & 84.95 & 6.97 \\
3480.919038 & 93.96 & 7.11 \\
3482.891047 & 80.4 & 7.21 \\
\hline
\end{tabular}

Notes.

${ }^{\text {a }}$ Errors as modeled; no additional noise error to be added.

(This table is available in its entirety in a machine-readable form in the online journal. A portion is shown here for guidance regarding its form and content.)

\subsubsection{Lick Radial Velocity Observation Adjustments}

For our study of $v$ Andromedae (McArthur et al. 2010), Fischer and Giguere forwarded us re-reduced velocities for $v$ Andromedae from Lick Observatory. They had improved the pipeline and included additional $\gamma$ offsets that were related to the use of different detectors on the instrument (D. Fischer \& M. Giguere 2009, private communication). The earlier Lick data for $v$ Andromedae (Wright et al. 2009) contained a signal at 3811 days which was not present in their re-reduced $\gamma$ adjusted data set as shown in Figure 1. This instrumental signal in the data created by neglected $\gamma$ offsets was identified as a fourth planet with period of 3848 days and possibly in resonance with $v$ Andromedae $\mathrm{c}$ in Curiel et al. (2011). Since the time span of the observations of the system was less than 1.5 cycles of this long-period signal, the instrumental effect on the data set was not revealed. In a Bayesian analysis of the separate reductions of the Lick velocities for $v$ Andromedae (Fischer et al. 2003; Wright et al. 2009), Tuomi et al. (2011) concluded that there were large inconsistencies (700 days) in the period determination of the proposed $v$ Andromedae e, based upon which Lick reduction was used in the analysis, which caused them to question the source of the fourth signal (bias or Keplerian signal).

While re-reduction of the Lick library is forthcoming (D. Fischer 2010, private communication) it has not been published at the time of this investigation so we included four independent $\gamma$ offsets in our fitting of the HD 128311 velocities that related to the timing of the detector changes. These $\gamma$ offsets and the time periods and number of observations in the fit (of the $\gamma$ offset) in the Lick velocities are shown in Table 5.

Additionally, we also considered two independent reductions of the Lick velocities - the set in Butler et al. (2006) and the set in Meschiari et al. (2009). When we compared the velocities, using only the data after the last Lick detector change (postJulian Date 2452515), we found a small yearly signal difference (as a function of day of the year) between the two sets shown 


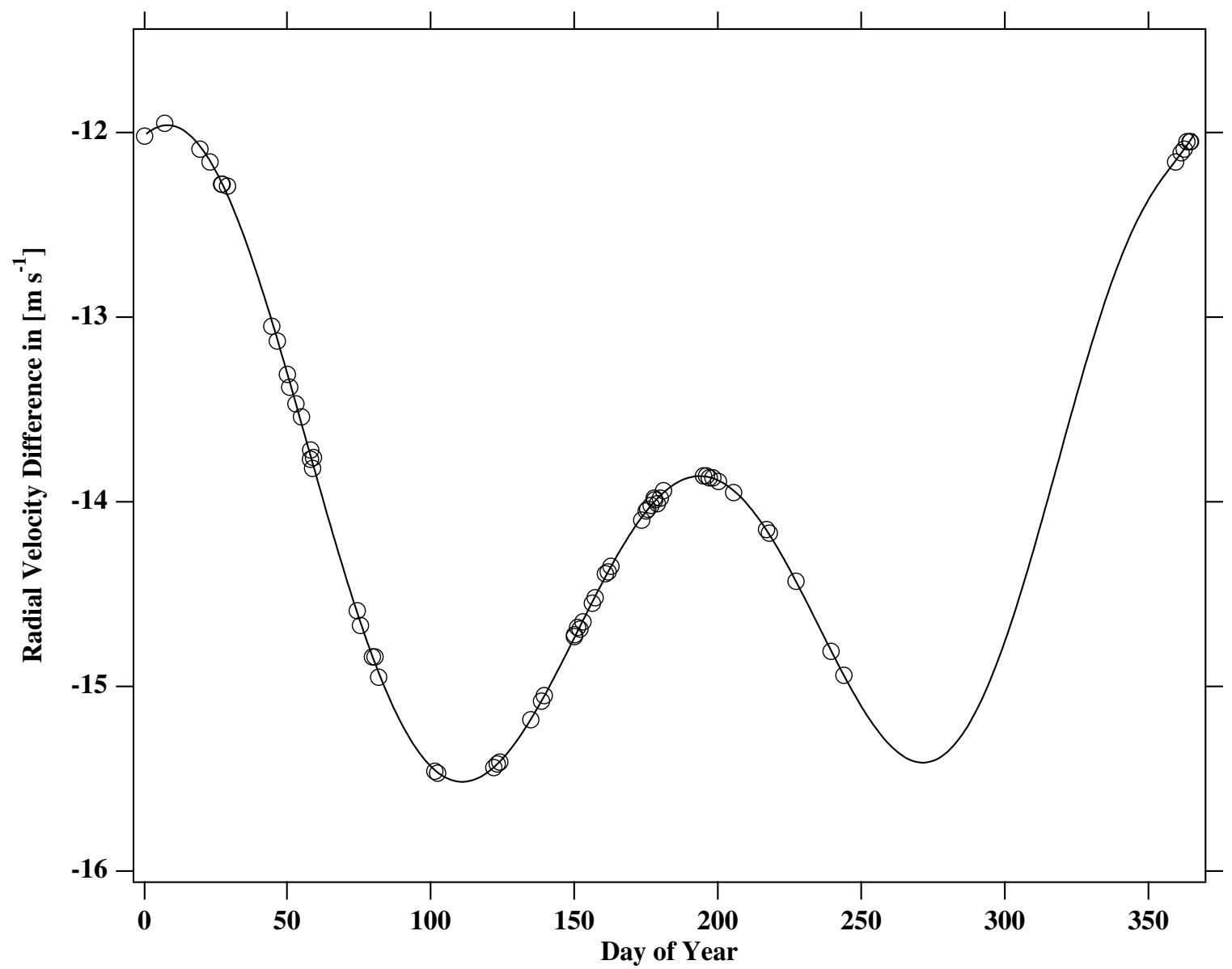

Figure 2. RV difference in the two reductions (Butler et al. 2006; Meschiari et al. 2009) of Lick HD 128311 data phased to 1 yr are shown by the open circles. The solid line is a polynomial that was fit to the difference and applied to the newer data set.

Table 5

$\gamma$ Offsets for Lick Radial Velocities of HD 128311

\begin{tabular}{lcccc}
\hline \hline $\begin{array}{l}\text { Julian Date } \\
\text { (Start) }\end{array}$ & $\begin{array}{c}\text { Julian Date } \\
\text { (End) }\end{array}$ & $\begin{array}{c}\text { Number } \\
\text { of Velocities }\end{array}$ & $\gamma$ & \pm Error \\
\hline 2450983 & 2451371 & 4 & 0.85 & 2.44 \\
2451409 & 2452163 & 14 & 4.80 & 5.34 \\
2452333 & 2452489 & 9 & 1.16 & 3.60 \\
2452515 & $2454602^{\mathrm{a}}$ & 64 & 3.44 & 1.19 \\
\hline
\end{tabular}

Note. ${ }^{a}$ Observation date of last Lick velocity included in this analysis, not necessarily the end date for this $\gamma$ value.

in Figure 2. We fit this difference with a polynomial, and adjusted the more recent data set (Meschiari et al. 2009) with the polynomial. We found a slightly reduced $\chi^{2}$ with the more recent (Meschiari et al. 2009) data set adjusted with the polynomial, and used this set of data in our solution.

\subsection{HST Astrometry Observations}

The astrometric observations were made with the HST FGS 1r, a two-axis interferometer, in position (POS) "fringetracking" mode. ${ }^{8}$ The FGS instrument is described in Nelan (2007). Benedict et al. (2007) describes the reduction and calibration of the data. All FGS data was calibrated with a new improved Optical Field Angle Distortion (OFAD) derived by

\footnotetext{
8 A detailed Instrument Handbook can be found on the Space Telescope Science Institute Web site:

http://www.stsci.edu/hst/fgs/documents/instrumenthandbook/.
}

McArthur, which is not yet unpublished, but available with the reduction pipeline. The astrometric data used in this research are available from the HST Program Schedule and Information Web site, ${ }^{9}$ in proposal numbers 11210 and 11788 . The two part pipeline used to reduce the raw data to the values used in this modeling is available with the latest calibration parameters from the Space Telescope Science Institute in IRAF STSDAS and in a stand-alone version available from one of the coauthors, the HST FGS Instrument Scientist at STSCI Ed Nelan.

FGS astrometric data are obtained by download from the HST online archival retrieval system and then processed through the FGS pipeline system. The initial calibration pipeline extracts the astrometry measurements (typically 1-2 minutes of fringe position information acquired at a $40 \mathrm{~Hz}$ rate, which yields several thousand discrete measurements) and calculates the median (after outlier removal) and performs error estimation. The second calibration stage applies the time-variant OFAD, corrects the velocity aberration, processes the time tags, and uses the JPL Earth orbit predictor (Standish 1990) to calculate the parallax factors. The form of the OFAD calibration is discussed in several calibration papers (McArthur et al. 1997, 2002, 2006). Ongoing stability tests (LTSTABs) are used to maintain this calibration. Instrumental systematics in FGS 1r (such as intraorbit drift and color and filter effects) and their corrections are discussed in later sections. After $20 \mathrm{yr}$ of calibration we have not found additional systematics in our data at a level that is higher than our detection limit. With the exception of

\footnotetext{
9 http://www.stsci.edu/hst/scheduling/program_information
} 


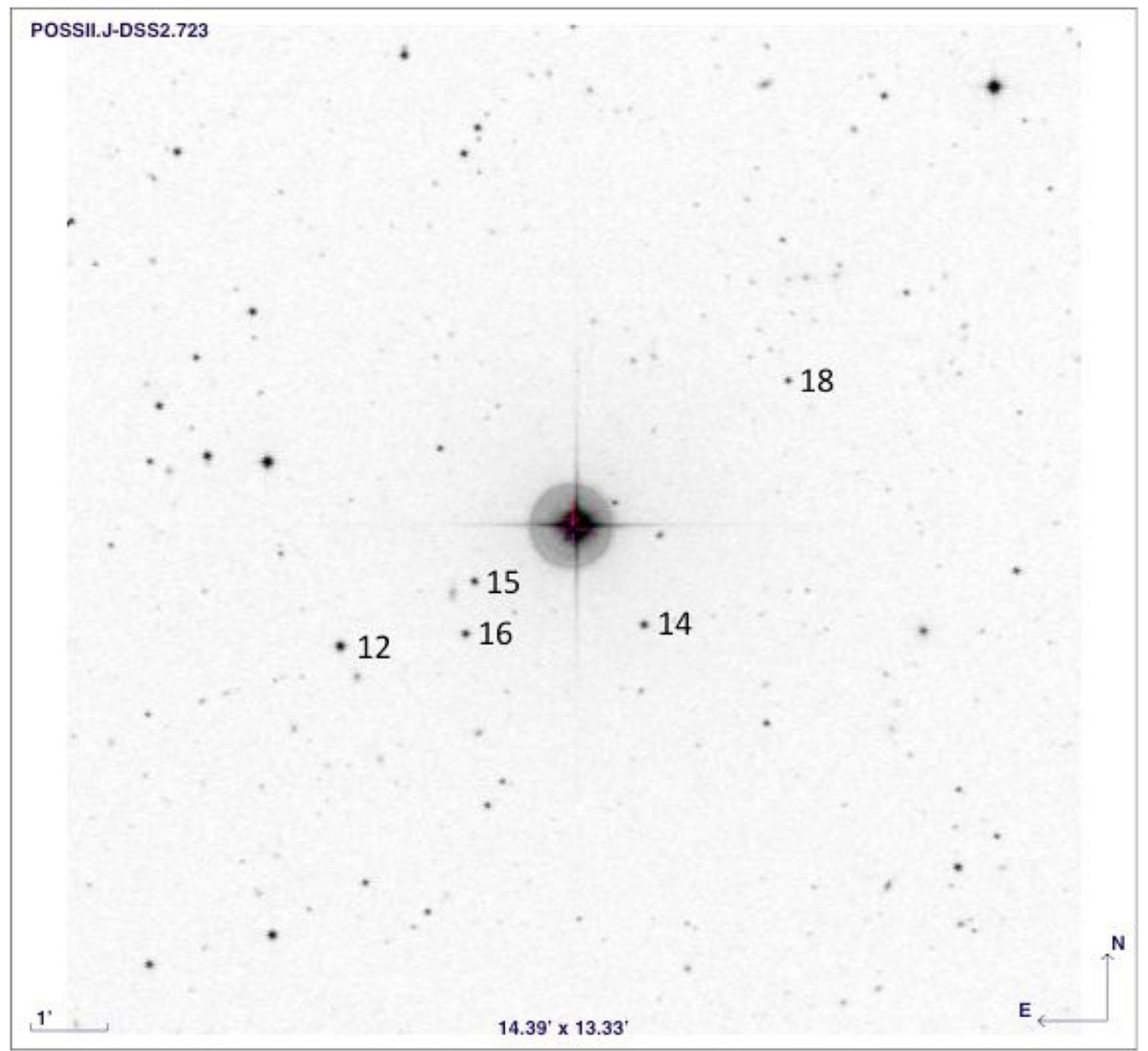

Figure 3. HD 128311 field with astrometric reference stars marked. The stars are listed in Table 11. The DSS image was secured using Aladin.

(A color version of this figure is available in the online journal.)

the Pleiades, regression analysis between Hipparcos and HST parallax measurements has shown not only good agreement between the determined parallaxes but an overestimation of error in HST astrometric measurements (Benedict et al. 2007; McArthur et al. 2010).

Twenty-nine orbits of HST astrometric observations were made between 2007 December and 2009 August. Because of observing constraints, there was a 225 day gap in observations between 2008 May and 2009 January. Every orbit contains several observations of HD 128311 and surrounding reference stars. The distribution of the reference stars in the HD 128311 field is shown in the Digital Sky Survey (DSS) image in Figure 3. The FGS measures the position of each star sequentially. Each epoch contains multiple visits, alternating between the target (HD 128311) and the five reference stars, providing $x(t)$ and $y(t)$ positions in the HST reference frame in seconds of arc. Observations during an orbit were corrected for slow FGS intraorbit positional drift by using an adaptable polynomial fitting routine amplified to model the high intra-orbit drift seen in some of these observations. Because HD 128311 is a bright star $(V=7.39)$ a neutral density filter (F5ND) was used. For the reference stars the $F 583 \mathrm{~W}$ filter was used. The dates of observation, the number of measurements of HD 128311 for each date, the HST orientation angles and the number of plate parameters are listed on Table 6. The HST astrometric data for HD 128311 and its reference stars is available only online (Table 7). For the most current calibration the data should be retrieved from the HST online archival retrieval system and processed through the two stage pipeline system.

\subsection{Spectrophotometric Parallaxes for the Reference Stars}

An HST target parallax is relative with respect to its reference frame. To go from relative to absolute parallax for HD 128311, we can either apply a statistically derived correction from relative to absolute parallax (van Altena et al. 1995; compare to Yale Parallax Catalog, YPC95), or use a Bayesian approach in which we use the derived spectroscopic parallaxes of the reference frame stars as input to the model. We estimate the absolute parallaxes of the reference frame stars from the colors, spectral type, and luminosity classes of the stars. We require the absolute magnitude, $M_{V}$, and $V$-band absorption, $A_{V}$ for the equation

$$
\pi_{\mathrm{abs}}=10^{-\left(V-M_{V}+5-A_{V}\right) / 5} .
$$

Our model then produces an absolute, not relative parallax. 
Table 6

HST Astrometric Observations of HD 128311 and the Reference Frame

\begin{tabular}{|c|c|c|c|c|c|}
\hline Orbit & Year & Day & Julian Date & $N_{\text {obs }}$ & HST Roll \\
\hline 1 & 2007 & 360 & 2454461.32336805 & 3 & 238.7345 \\
\hline 2 & 2007 & 364 & 2454464.51170138 & 4 & 239.3361 \\
\hline 3 & 2008 & 6 & 2454471.50753472 & 4 & 239.7188 \\
\hline 4 & 2008 & 25 & 2454491.22082175 & 4 & 238.9152 \\
\hline 5 & 2008 & 32 & 2454498.41331018 & 4 & 239.9798 \\
\hline 6 & 2008 & 38 & 2454503.80822916 & 4 & 256.6916 \\
\hline 7 & 2008 & 43 & 2454509.26917824 & 4 & 258.6858 \\
\hline 8 & 2008 & 49 & 2454514.59637731 & 4 & 261.1828 \\
\hline 9 & 2008 & 55 & 2454520.52322916 & 4 & 264.1754 \\
\hline 10 & 2008 & 62 & 2454528.38375000 & 4 & 265.9971 \\
\hline 11 & 2008 & 68 & 2454534.31335648 & 4 & 265.9971 \\
\hline 12 & 2008 & 76 & 2454542.23754629 & 4 & 276.9977 \\
\hline 13 & 2008 & 83 & 2454548.69702546 & 4 & 276.9977 \\
\hline 14 & 2008 & 87 & 2454553.36010416 & 4 & 276.9977 \\
\hline 15 & 2008 & 103 & 2454569.14370370 & 4 & 330.2641 \\
\hline 16 & 2008 & 121 & 2454587.06107639 & 4 & 335.0302 \\
\hline 17 & 2008 & 131 & 2454597.11729166 & 4 & 20.03228 \\
\hline 18 & 2008 & 140 & 2454605.84104166 & 4 & 24.03138 \\
\hline \multicolumn{6}{|c|}{ Servicing mission } \\
\hline 19 & 2009 & 1 & 2454832.61401620 & 4 & 235.9564 \\
\hline 20 & 2009 & 38 & 2454870.30037037 & 4 & 256.7992 \\
\hline 21 & 2009 & 46 & 2454877.82504629 & 4 & 238.9172 \\
\hline 22 & 2009 & 65 & 2454896.60428240 & 4 & 268.9989 \\
\hline 23 & 2009 & 83 & 2454915.25145833 & 4 & 268.9952 \\
\hline 24 & 2009 & 96 & 2454928.03842592 & 4 & 283.9783 \\
\hline 25 & 2009 & 117 & 2454949.01655092 & 4 & 340.0253 \\
\hline 26 & 2009 & 163 & 2454994.56111111 & 4 & 31.02961 \\
\hline 27 & 2009 & 190 & 2455022.05805555 & 4 & 79.0068 \\
\hline 28 & 2009 & 204 & 2455036.44101851 & 4 & 81.00542 \\
\hline 29 & 2009 & 222 & 2455053.81746527 & 4 & 81.00542 \\
\hline
\end{tabular}

\subsubsection{Reference Star Photometry}

Our bandpasses for reference star photometry include: BV (CCD photometry from a $1 \mathrm{~m}$ telescope at New Mexico State University), ${ }^{10} V$ (from FGS 1r), and $J H K$ from Two Micron All Sky Survey. ${ }^{11}$ The $J H K$ values have been transformed to

\footnotetext{
10 The Apache Point Observatory $3.5 \mathrm{~m}$ telescope is owned and operated by the Astrophysical Research Consortium.

11 The Two Micron All Sky Survey is a joint project of the University of Massachusetts and the Infrared Processing and Analysis Center/California Institute of Technology.
}

the Bessell \& Brett (1988) system using the transformations provided in Carpenter (2001). Table 8 lists VJHK photometry for the target and reference stars indicated in Figure 3. Figure 4 contains a $(J-K)$ versus $(V-K)$ color-color diagram with reference stars and HD 128311 labeled.

\subsubsection{Spectroscopy, Luminosity Class, and Reduced Proper Motion}

The derived absolute magnitudes are critically dependent on the assumed stellar luminosities, a parameter impossible to obtain for all but the latest type stars using only Figure 4. The spectra from which we estimated spectral type and luminosity class come from the Cerro Tololo Inter-American Observatory $4 \mathrm{~m}$ with RCSPEC. The KPGL1-1 grating was used in first order, which has $6321 \mathrm{~mm}^{-1}$, blaze wavelength $4200 \AA$ and a $150 \mu \mathrm{m}$ slit $\left(=1^{\prime \prime}\right)$. The dispersion was $1.0 \AA$ pixel $^{-1}$ with wavelength coverage from 3500 to $5850 \AA$. Classifications used a combination of a large library of MK templates for matching and line ratios. The faintest target (REF-6) had about 4000 peak counts in the continuum above sky per pixel, or signalto-noise ratio $(\mathrm{S} / \mathrm{N}) \sim 60$. While the $\mathrm{S} / \mathrm{N}$ was very high and the matching library substantial, we found that the resulting instrumental resolution limited our spectral classification to \pm 2 subclasses. The field had very low extinction $A_{v} \leqslant 0.1$ for all stars.

To confirm the luminosity classes, we obtain PPMXL proper motions (Roeser et al. 2010) for a one-degree-square field centered on HD 128311 shown in Figure 5, and then iteratively employ the technique of reduced proper motion (Yong \& Lambert 2003; Gould \& Morgan 2003) to discriminate between giants and dwarfs.

\subsubsection{Estimated Reference Frame Absolute Parallaxes}

We derive spectrophotometric parallaxes using our estimated spectral types and luminosity class and $M_{V}$ values from Cox (2000). Our adopted input errors for distance moduli, $(m-M)_{0}$, are $0.4 \mathrm{mag}$ for all reference stars. Contributions to the error are a small but undetermined $A_{V}$ and in $M_{V}$ errors due to uncertainties in color to spectral type mapping. All reference star absolute parallax estimates, spectral types and luminosities are listed in Table 9. Individually, no reference star absolute parallax is better determined than $\left(\sigma_{\pi} / \pi\right)=18 \%$. The average input absolute parallax for the reference frame is $\left\langle\pi_{\mathrm{abs}}\right\rangle=0.7 \mathrm{~ms}$ of arc (mas), a quantity known to $\sim 40 \%$ (standard deviation

Table 7

HST Astrometric Data for HD 128311 and Reference Stars

\begin{tabular}{lcccccccc}
\hline \hline Set & ObsID & Star & rollV3 & Filter & Xmad & Ymad & Xvaofadapj2d & Yvaofadapj2d \\
\hline 1 & F9YM1902M & 16 & 238.7345 & F583W & 0.00295 & 0.0024 & -112.8103 & 755.9297 \\
\hline
\end{tabular}

(This table is available in its entirety in a machine-readable form in the online journal. A portion is shown here for guidance regarding its form and content.)

Table 8

$V$ and Near-IR Photometry of HD 128311 and Reference Stars

\begin{tabular}{lccccc}
\hline \hline ID & $V$ & $K$ & $(J-H)$ & $(J-K)$ & $(V-K)$ \\
\hline 2 & $7.48 \pm 0.03$ & $5.142 \pm 0.017$ & $0.469 \pm 0.036$ & $0.630 \pm 0.025$ & $2.338 \pm 0.0345$ \\
12 & $14.15 \pm 0.03$ & $12.635 \pm 0.026$ & $0.280 \pm 0.040$ & $0.385 \pm 0.037$ & $1.515 \pm 0.040$ \\
14 & $15.45 \pm 0.03$ & $13.92 \pm 0.063$ & $0.297 \pm 0.055$ & $0.333 \pm 0.072$ & $1.53 \pm 0.070$ \\
15 & $15.62 \pm 0.03$ & $14.056 \pm 0.06$ & $0.375 \pm 0.051$ & $0.388 \pm 0.069$ & $1.564 \pm 0.067$ \\
16 & $15.29 \pm 0.03$ & $13.466 \pm 0.045$ & $0.368 \pm 0.042$ & $0.430 \pm 0.054$ & $1.824 \pm 0.054$ \\
18 & $16.5 \pm 0.03$ & $14.955 \pm 0.133$ & $0.373 \pm 0.091$ & $0.429 \pm 0.147$ & $1.545 \pm 0.136$ \\
\hline
\end{tabular}




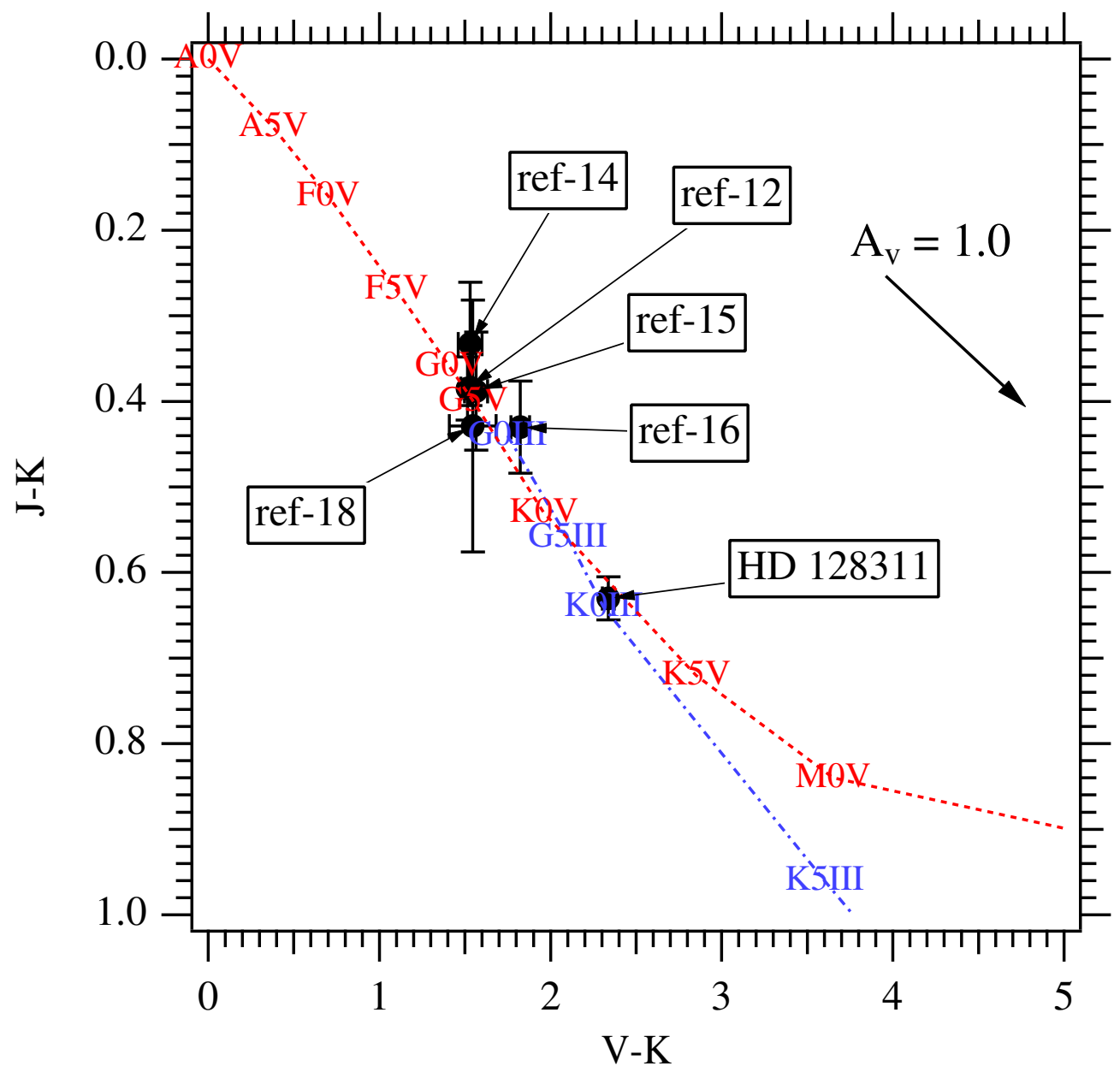

Figure 4. $(J-K)$ vs. $(V-K)$ color-color diagram for stars in the HD 128311 field identified in Table 8 . The dashed line is the locus of dwarf (luminosity class V) stars of various spectral types; the dot-dashed line is for giants (luminosity class III). The reddening vector indicates $A_{V}=1.0$ for the plotted color systems. Along this line of sight maximum extinction is $A_{V} \sim 0.3$ (Schlegel et al. 1998).

(A color version of this figure is available in the online journal.)

Table 9

Astrometric HD 128311 Reference Star Adopted Spectrophotometric Parallaxes

\begin{tabular}{lcccccc}
\hline \hline ID & Sp. T. $^{\text {a }}$ & $V$ & $B-V$ & $M_{v}$ & $A_{v}$ & $\begin{array}{c}\pi_{\text {abs }} \\
(\mathrm{mas})\end{array}$ \\
\hline 12 & G0V & 14.15 & $0.59 \pm 0.03$ & 4.20 & 0.07 & $1.066 \pm 0.267$ \\
14 & G1V & 15.45 & $0.61 \pm 0.07$ & 4.24 & 0.06 & $0.594 \pm 0.148$ \\
15 & G1V & 15.62 & $0.54 \pm 0.08$ & 4.24 & 0.06 & $0.553 \pm 0.138$ \\
16 & G4V & 15.29 & $0.69 \pm 0.07$ & 4.82 & 0.10 & $0.882 \pm 0.220$ \\
18 & G1V & 16.50 & $0.61 \pm 0.11$ & 4.24 & 0.10 & $0.371 \pm 0.093$
\end{tabular}

Note. ${ }^{\text {a }}$ Spectral types and luminosity class estimated from colors and reduced proper motion diagram.

of the mean of five reference stars). We compare this to the correction to absolute parallax discussed and presented in YPC95 (Section 3.2, Figure 3). Entering YPC95, Figure 3, with the Galactic latitude of HD 128311, $b=+59.85$, and average magnitude for the reference frame, $\left\langle V_{\text {ref }}\right\rangle=15.4$, we obtain a correction to absolute parallax of 1.3 mas, with is higher than our average input parallax, but derived from a compressed portion of the YPC graph. Nonetheless, rather than apply a model-dependent correction to absolute parallax, we introduce our spectrophotometrically estimated reference star parallaxes into our reduction model as observations with error.
Table 10

Astrometric HD 128311 Reference Star Proper Motions

\begin{tabular}{lrrrrr}
\hline \hline & \multicolumn{2}{c}{ Input (PPMXL) } & & \multicolumn{2}{c}{ Final (HST) } \\
\cline { 2 - 3 } \cline { 5 - 5 } ID & \multicolumn{1}{c}{$\mu_{\alpha}{ }^{\mathrm{a}}$} & \multicolumn{1}{c}{$\mu_{\delta}{ }^{\mathrm{a}}$} & & $\mu_{\alpha}$ & \multicolumn{1}{c}{$\mu_{\delta}$} \\
\hline 12 & $-6.2 \pm 3.6$ & $-6.0 \pm 3.6$ & & $-8.17 \pm 0.38$ & $-8.59 \pm 0.34$ \\
14 & $-4.5 \pm 3.6$ & $-5.2 \pm 3.6$ & & $-5.02 \pm 0.37$ & $-6.12 \pm 0.33$ \\
15 & $-7.5 \pm 3.6$ & $4.4 \pm 3.6$ & & $-7.79 \pm 0.39$ & $8.28 \pm 0.34$ \\
16 & $54.3 \pm 3.6$ & $-5.1 \pm 3.6$ & & $-52.08 \pm 0.33$ & $-7.72 \pm 0.27$ \\
18 & $3.0 \pm 3.8$ & $3.6 \pm 3.8$ & & $2.92 \pm 0.85$ & $1.53 \pm 0.71$ \\
\hline
\end{tabular}

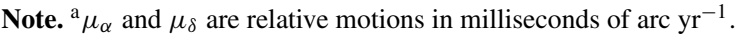

\subsubsection{Estimated Reference Frame Proper Motions}

Typically, we use proper motion values from UCAC3 (Zacharias et al. 2009) or the PPMXL catalog (Roeser et al. 2010) as observations with error in the model. For this field we find better observational fits with the PPMXL catalog data, with a vector offset in the UCAC 3 catalog. The PPMXL proper motions, the reference frame derived values and our final model values are found in Table 10.

\subsection{Astrometric Model}

The HD 128311 reference frame contained five reference stars plus HD 128311. HST rolls with the observations, so 


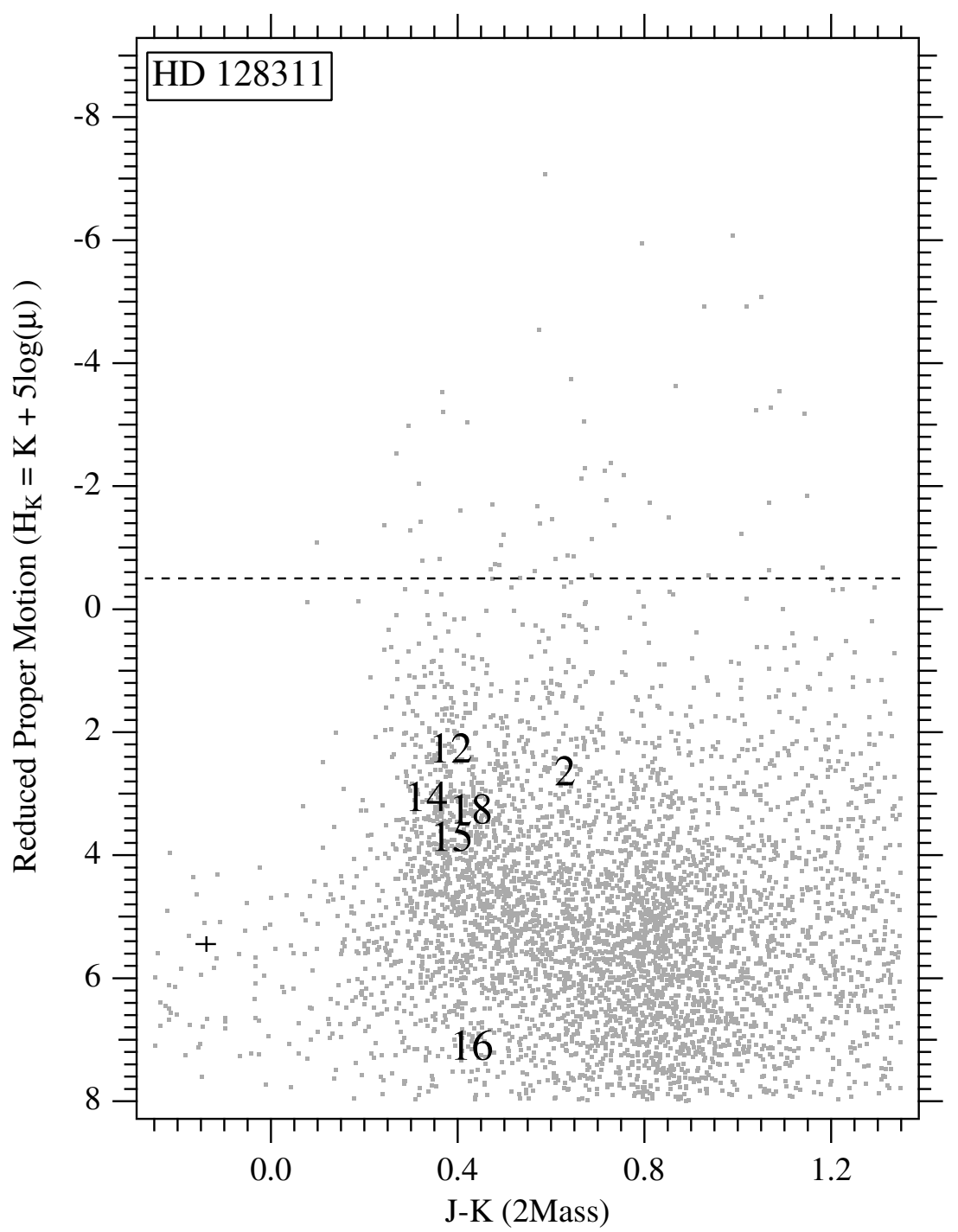

Figure 5. Reduced proper motion diagram for 5800 stars in a $1^{\circ}$ field centered on HD 128311. Star identifications are in Table 8. For a given spectral type, giants and sub-giants have more negative $H_{K}$ values and are redder than dwarfs in $(J-K) . H_{K}$ values are derived from "final" proper motions in Table 10 . The small cross at the lower left represents a typical $(J-K)$ error of $0.04 \mathrm{mag}$ and $H_{K}$ error of $0.17 \mathrm{mag}$. The horizontal dashed line is a giant-dwarf demarcation derived from a statistical analysis of the Tycho input catalog (D. R. Ciardi 2004, private communication).

the positions $\left(x^{\prime}, y^{\prime}\right)$ of the target and reference stars change with each observation set. We use an overlapping plate model that includes parameters for scale, rotation, and offset. Each plate expresses its relation to the constraint or master plate, usually one of the central observations, with these parameters. The astrometric model also accounts for the movements of each star, which are time-dependent, given by the absolute parallax $\pi_{\text {abs }}$ and the proper motion components, $\mu_{\alpha}$ and $\mu_{\delta}$. We include instrumentally caused position shift parameters for the crossfilter and lateral color. The standard coordinate catalog positions $\xi$ and $\eta$ are the result of modeling these equations of condition:

$$
\begin{gathered}
x^{\prime}=x+l c_{x}(B-V)-\Delta \mathrm{XF} x, \\
y^{\prime}=y+l c_{x}(B-V)-\Delta \mathrm{XF} y, \\
\xi=A x^{\prime}+B y^{\prime}+C-\mu_{\alpha} \Delta t-P_{\alpha} \pi-\mathrm{ORBIT}_{x}, \\
\eta=-B x^{\prime}+A y^{\prime}+F-\mu_{\delta} \Delta t-P_{\delta} \pi-\mathrm{ORBIT}_{y},
\end{gathered}
$$

where $x$ and $y$ are the measured coordinates from HST; $l c_{x}$ and $l c_{y}$ are the lateral color corrections, and $B-V$ are the $B-V$ colors of each star; $\triangle \mathrm{XF} x$ and $\triangle \mathrm{XFy}$ are the cross-filter corrections in $x$ and $y$, applied only to the observations of HD 128311. A and $B$ are scale and rotation plate constants, and $C$ and $F$ are offsets, $\mu_{\alpha}$ and $\mu_{\delta}$ are proper motions, $\Delta t$ is the epoch difference from the mean epoch, $P_{\alpha}$ and $P_{\delta}$ are parallax factors, and $\pi$ is the parallax. We obtain the parallax factors from a JPL Earth orbit predictor Standish (1990), upgraded to version DE405. ORBIT is a function using Thiele-Innes constants (Heintz 1978) of the traditional astrometric and RV orbital elements. We used a model written in the GaussFit language (Jefferys et al. 1988) employing robust estimation to derive a simultaneous solution. Table 11 shows the resulting astrometric catalog from the combined orbital modeling.

Additionally, equations of condition relate an initial and final parameter value. For the reference stars there are equations in the model for proper motion and spectrophotometric parallax. For the target star (HD 128311) we add equations for crossfilter. Both target and reference stars have equations for lateral color parameters. The roll of the constraint plate also has a condition equation. Through these additional equations of condition the $\chi^{2}$ minimization process is allowed to adjust 
Table 11

Astrometric Catalog of HD 128311 and Reference Stars

\begin{tabular}{lccccccc}
\hline \hline Star & $\begin{array}{c}\text { Mag } \\
V\end{array}$ & $\begin{array}{c}\text { R.A. }^{\mathrm{a}} \\
(\mathrm{deg})\end{array}$ & $\begin{array}{c}\text { Decl. } \\
(\mathrm{deg})\end{array}$ & $\begin{array}{c}\xi^{\mathrm{b}} \\
(\operatorname{arcsec})\end{array}$ & $\begin{array}{c}\sigma_{\xi} \\
(\operatorname{arcsec})\end{array}$ & $\begin{array}{c}\eta \\
(\operatorname{arcsec})\end{array}$ & $\begin{array}{c}\sigma_{\eta} \\
(\operatorname{arcsec})\end{array}$ \\
\hline HD 128311 & 7.39 & 219.00281 & 9.74596 & -1.11484 & 0.00011 & 731.65337 & 0.00011 \\
12 & 14.15 & 219.05321 & 9.72182 & 151.39824 & 0.00019 & 859.97773 & 0.00020 \\
14 & 15.44 & 218.98751 & 9.72575 & 43.39602 & 0.00017 & 652.54731 & 0.00017 \\
15 & 15.58 & 219.02412 & 9.73521 & 64.64370 & 0.00019 & 785.18893 & 0.00021 \\
16 & 15.3 & 219.02604 & 9.72411 & 104.17150 & 0.00016 & 774.82462 & 0.00015 \\
18 & 16.54 & 218.95594 & 9.77737 & -171.82461 & 0.00050 & 625.47534 & 0.00051
\end{tabular}

Notes.

a Predicted coordinates for equinox J2000.0.

${ }^{b}$ Relative coordinates in the reference frame of the constrained plate (set 18 , with roll $=24.03138$ ).

parameter values by amounts constrained by the input errors. In this quasi-Bayesian approach prior knowledge is input as an observation with associated error, not as a hardwired quantity known to infinite precision. For HD 128311 no priors were used for parallax or proper motion or the orbital motion.

\subsubsection{Astrometric Reference Frame Residual Assessment}

The reference frame stars are modeled without HD 128311 many times to assess plate models, prior knowledge of spectrophotometric parallaxes, catalog proper motions, and stability as a reference star. We plotted reference frame $x$ and $y$ residuals against a number of spacecraft, instrumental, and astronomical parameters. These included $x$ and $y$ position within our total field of view, radial distance from the field-of-view center, reference star $V$ magnitude and $B-V$ color, and epoch of observation. We saw no trends indicating systematic instrumental effects that were larger than 1 mas. There is a predicted small upward trend in residuals with the faintest star.

\subsubsection{Astrometric Catalog Residual Assessment}

HST FGS 1r has raw distortions of more than $1^{\prime \prime}$, but the OFAD (McArthur et al. 2002) calibration reduces these distortions to below 2 mas over much of the FGS 1r field. Histograms of the astrometric residuals (Figure 6) show the goodness of the fit. The astrometric catalog shown in Table 11 has $\xi$ and $\eta$ standard coordinates determined with average position errors $\left\langle\sigma_{\xi}\right\rangle=0.13$ and $\left\langle\sigma_{\eta}\right\rangle=0.11$ mas. The median average deviations of these positions are $\xi_{\mathrm{MAD}}=0.066$ and $\eta_{\mathrm{MAD}}=0.056$ mas.

\subsection{Radial Velocity Model}

We model the radial $(\hat{z})$ motion of the stellar orbital movement around the barycenter of the HD 128311 system. The equation used is a projection of a Keplerian orbital velocity to observer's line of sight plus a constant velocity offset:

$$
\begin{aligned}
\cos v w= & \frac{\cos (E)-e}{1-(e \times \cos (E))} \times \cos (\omega) \\
& -\frac{\sqrt{1-e^{2}} \times \sin E}{1-(e \times \cos (E))} \times \sin (\omega), \\
Z=\gamma+ & K 1 \times(\text { ecc } \times \cos (\omega)+\cos v w),
\end{aligned}
$$

where $e$ is eccentricity, $\omega$ is the longitude of per astron, $K 1$ is the semi-amplitude of the RV signal, $E$ is the eccentric anomaly from Kepler's equation, and $\gamma$ is the constant velocity offset. We model three planetary orbits and five $\gamma$ 's, one for the HET and four for the Lick data.

\subsection{Combined Orbital Model}

For the combined orbital model, we use a linear combination of three unperturbed Keplerian orbits, which is an acceptable first-order approximation of the orbital elements, and we model one of these astrometrically (the other two orbital perturbations are below the detection limit of the HST FGS. Additionally, we constrain a relationship between the astrometry and the RV through this equation (Pourbaix \& Jorissen 2000):

$$
\frac{\alpha \sin i}{\pi_{\mathrm{abs}}}=\frac{P K\left(1-e^{2}\right)^{1 / 2}}{2 \pi \times 4.7405},
$$

where quantities only derivable from the astrometry (parallax, $\pi_{\mathrm{abs}}$, primary perturbation orbit size, $\alpha$, and inclination, $i$ ) are on the left, and quantities derivable from both (the period, $P$, and eccentricity, $e$ ), or RVs only (the RV amplitude of the primary, $K$ ), are on the right. The parallax, proper motion, and orbital elements were all the result of using the combined orbital model in which there was simultaneous modeling of the HST astrometry and RVs from Lick and the HET. Our long-term stability tests performs a full computation of perturbed orbits, which is shown in Section 4.5.

\section{RESULTS}

\subsection{HST Parallax and Proper Motions of HD 128311}

We find a parallax of $60.532 \pm 0.149$ mas for HD 128311 . This parallax lies between the parallaxes determined with the two solutions of the Hipparcos data, with an error five times lower (Perryman et al. 1997; van Leeuwen 2007). We find $\mu_{x}$ $=204.963 \pm 0.162$ and $\mu_{y}=-249.760 \pm 0.143$. These proper motions agree with Hipparcos and PPMXL values, but the HST motions have smaller uncertainties. HST proper motions are relative to the reference frame. No a priori values for proper motion and parallax were used as input for HD 128311, only for the reference frame. These results are shown in Table 12, including independently determined values for comparison.

\subsection{Orbital Solution}

\subsubsection{Radial Velocity Periodograms and Bootstrap Randomization}

Until this work, the most recent published orbital solution of HD 128311 was presented in Meschiari et al. (2009). It included newer Lick velocities and HET velocities taken from Wittenmyer et al. (2009). The best-fit Newtonian orbital parameters from this fit produces a system that loses stability in the first $1000 \mathrm{yr}$. We model these same Lick values but adjusted for additional $\gamma$ offsets (see Section 3.1.1) combined with our HET 


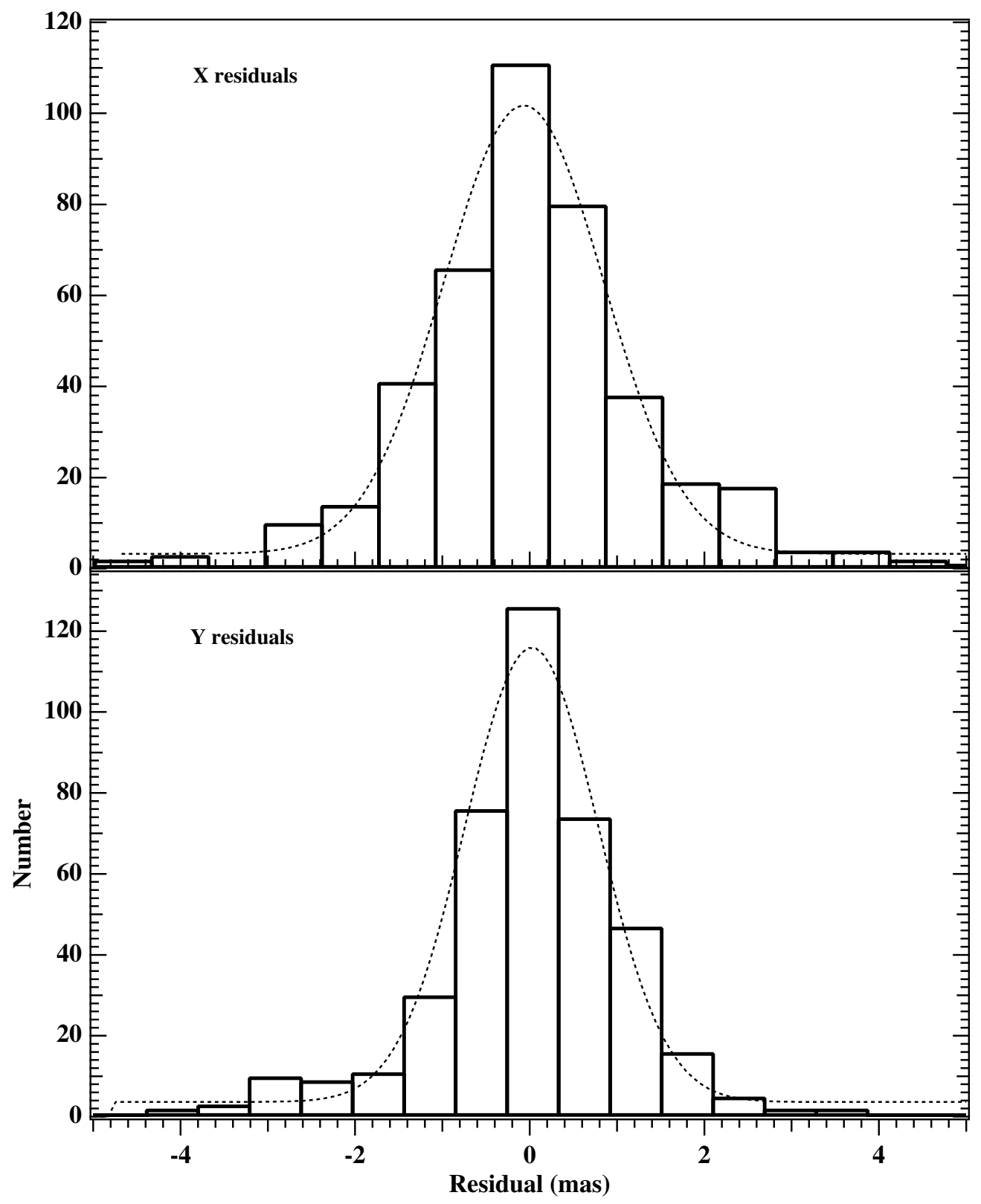

Figure 6. Histograms of the astrometric HD 128311 residuals of the fit.

Table 12

Parallax and Proper Motions of HD 128311

\begin{tabular}{lccc}
\hline \hline ID & Parallax & $\mu_{x}$ & $\mu_{y}$ \\
\hline$H S T$ & $60.532 \pm 0.149$ & $204.963 \pm 0.162$ & $-249.760 \pm 0.143$ \\
\hline HIP 2007 & $60.60 \pm 0.83$ & $204.74 \pm 0.74$ & $-249.98 \pm 0.61$ \\
HIP1997 $^{\mathrm{b}}$ & $60.35 \pm 0.99$ & $205.46 \pm 0.89$ & $-249.68 \pm 0.7$ \\
PPMXL $^{\mathrm{c}}$ & & $205.1 \pm 0.7$ & $-249.7 \pm 0.6$ \\
\hline
\end{tabular}

\section{Notes.}

a Perryman et al. (1997).

b van Leeuwen (2007).

${ }^{\mathrm{c}}$ Roeser et al. (2010).

velocities. We use additional velocities to those in Wittenmyer et al. (2009), and have reduced all of the HET data set with our own independent reduction pipeline (shown in Table 4), so the values used in this paper are different and distinct from those presented in Wittenmyer et al. (2009).
We analyzed weighted Lomb-Scargle periodograms of the residuals from the following three fits: (1) only $\gamma$ 's fitted, (2) $\gamma$ 's and planet b fitted, and (3) $\gamma$ 's and planets b and c fitted. We looked for evidence of additional planetary periodic signals in these periodograms (see Figure 7). In the periodogram of the residuals to a Keplerian model that contains the planets $b$ and $c$, we see a signal at $\sim 11.22$ days, and false alarm probability of $1.3 \times 10^{-40}$. A weighted Kepler periodogram (Zechmeister \& Kürster 2009) had a signal at $\sim 11.22$ days with a false alarm probability of $3.7 \times 10^{-65}$. Even though the period at $\sim 11.22$ days had very high power, we performed a bootstrap randomization of the data set. We randomly shuffled the data, fixing the observation times for 100,000 trials. We assessed these shuffles with the same weighted Lomb-Scargle period-finding program, and in the interval between 11 and 11.5 days the highest power in any of the 100,000 randomized data periodograms was a single peak with probability of $3.35 \times 10^{-04}$, well below that of the actual data which was $3.7 \times$ $10^{-65}$. After modeling the signal found at 11.22 days as a planet, 


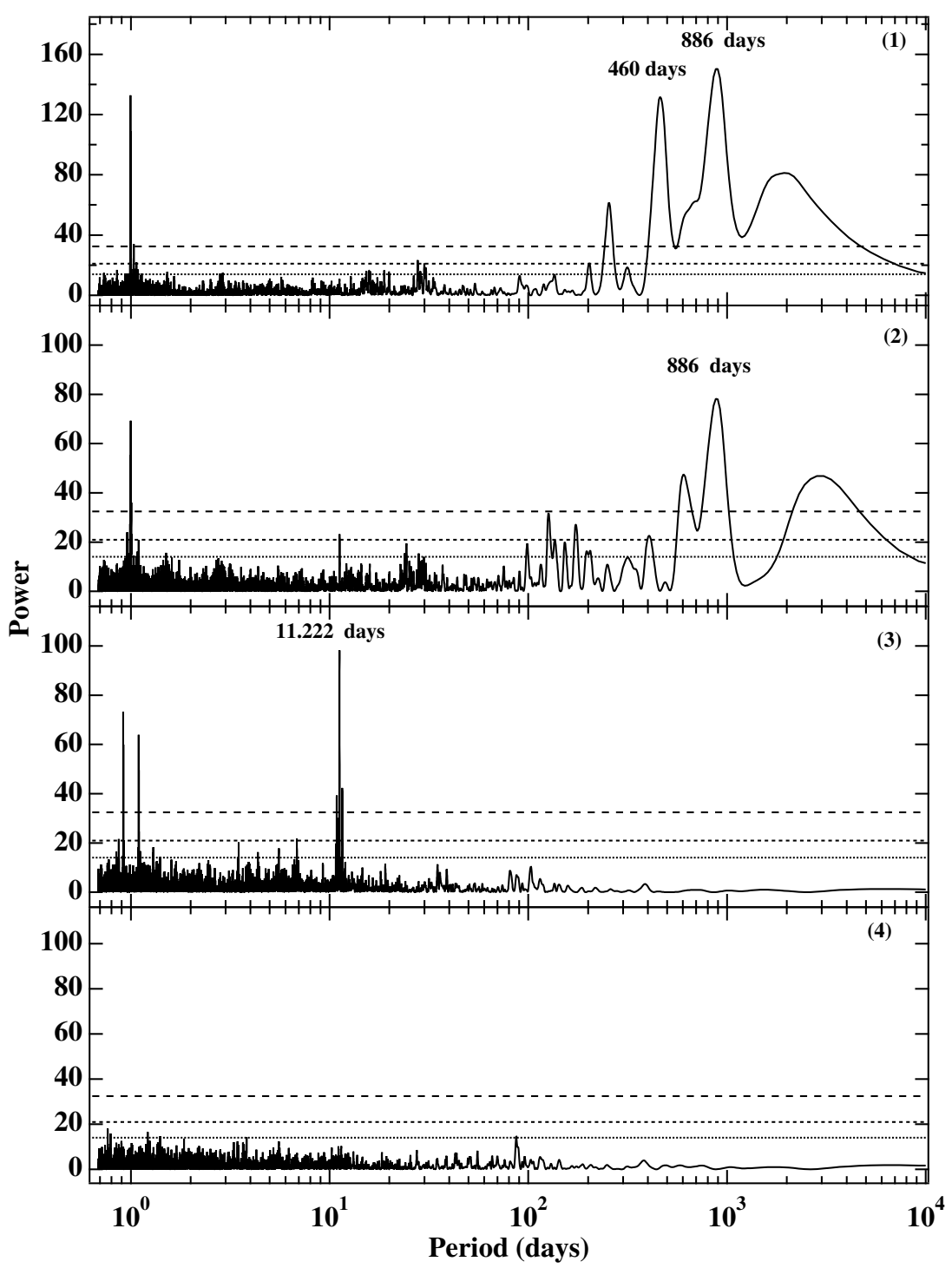

Figure 7. Periodogram of the radial velocity data of HD 128311 from two sources. Panel (1) shows peaks for planets b (460 days) and c (885 days). The false alarm probability of the peak at 460 days is $1.36 \times 10^{-53}$ and the peak at 885 days is $1.22 \times 10^{-61}$. Panel (2) shows the periodogram of the residuals of a Keplerian model that contains planet b; planet c (period 885 days) can be seen. The false alarm probability of the peak at 885 days is $9.2 \times 10^{-31}$. Panel (3) shows the periodogram of the residuals of a Keplerian model that contains planets b and c; possible planet d (11.222 days) emerges. The false alarm probability of the peak at 11.222 days is $1.49 \times 10^{-39}$. Panel (4) shows the periodogram of the residuals to a Keplerian model that contains planet b (period 460 days), planet $\mathrm{c}$ ( 885 days), and planet $\mathrm{d}$ (11.222 days). The dotted lines show the significance levels of the power in each plot, from the top: $1.0 \times 10^{-10}, 1.0 \times 10^{-5}$, and $1.0 \times 10^{-2}$.

Table 13

HD 128311 RV Modeling

\begin{tabular}{lrcc}
\hline \hline Model & $\chi^{2}$ & DOF & $\begin{array}{c}\mathrm{rms} \\
\left(\mathrm{m} \mathrm{s}^{-1}\right)\end{array}$ \\
\hline No planets $^{\mathrm{a}}$ & 37125 & 443 & 77.11 \\
$\mathrm{~b}$ & 9158 & 438 & 43.99 \\
$\mathrm{~b}+\mathrm{c}$ & 934 & 433 & 13.32 \\
$\mathrm{~b}+\mathrm{c}+\mathrm{d}$ & 437 & 428 & 9.37 \\
\hline
\end{tabular}

Note. ${ }^{\text {a }}$ Solve for $\gamma$ offsets only.

we find no other significant signals in the data set, as seen in panel (d). Finding no additional periodic signals to model from the periodograms in panel (d), we add a slope to the model to test for a longer period planet. We do not find a significant slope in this data set. Table 13 shows the $\chi^{2}$, degrees of freedom (DOFs) and rms of the various HD 128311 independent RV planet modeling.
We look at a comparison of panel (a) from Figure 7 to a perfect data set created from the orbital fit of Table 14 with the precise epochs of our observational data shown in Figure 8 to investigate the orbital periods. The periodogram of the perfect data sets displays many of the features of the observational data sets, including the strong signal around a period of one day. The window function shown in Figure 9 shows that the peak at one day is due to sampling.

\subsubsection{Simultaneous Radial Velocity and Astrometry Modeling}

A combined GaussFit model was used to simultaneously model the astrometry and RV observations. (A review of GaussFit being used for problems of linear regression with errors in both variables and the errors it produces is contained in Murtagh 1990.) Astrometric modeling of the target and reference frame consisted of scale, lateral color, cross-filter, proper motion, parallax, and the astrometric orbital elements of either planet $\mathrm{c}$ alone or planets $\mathrm{b}$ and $\mathrm{c}$, and was carried out as detailed 


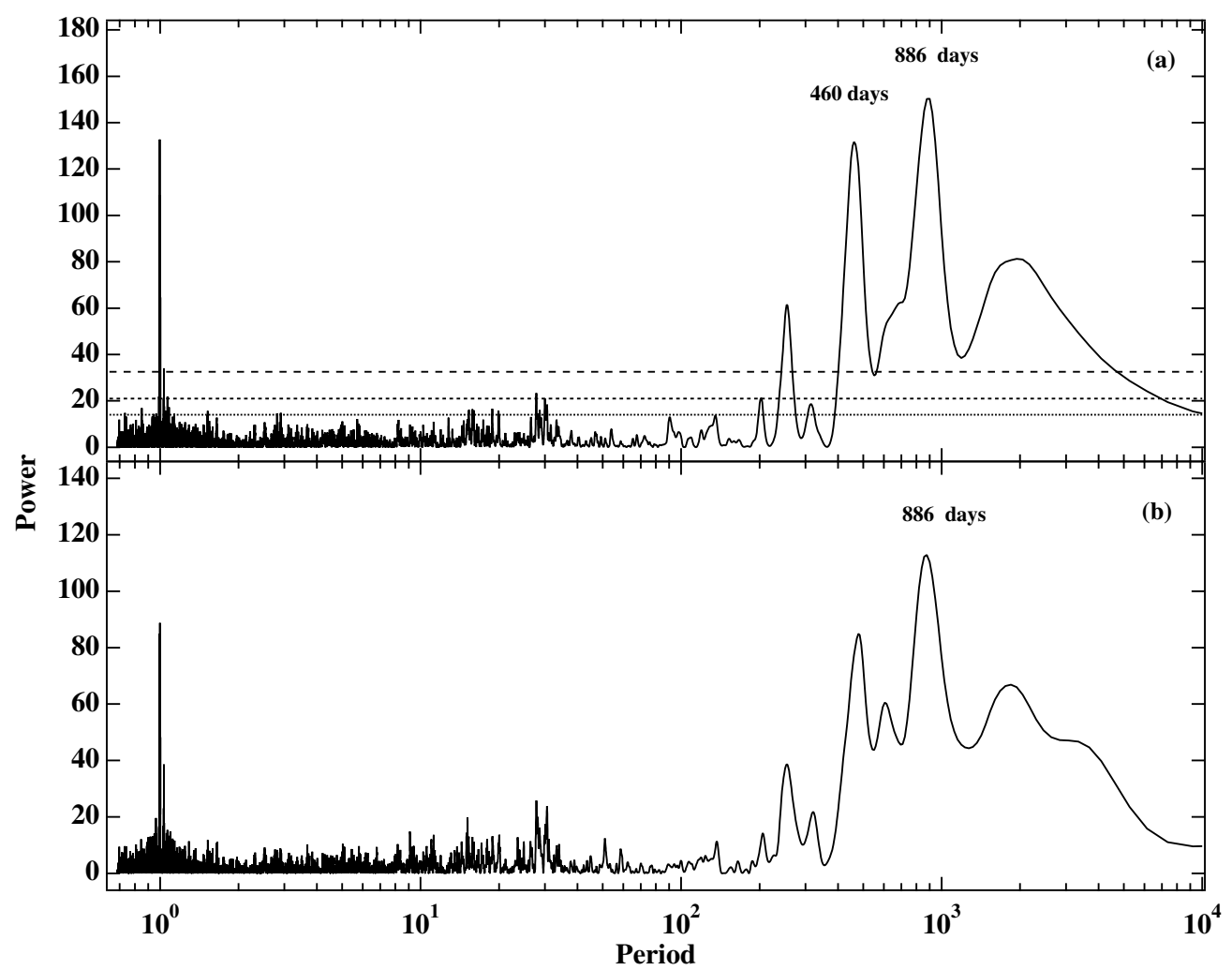

Figure 8. Periodogram of the radial velocity data of HD 128311 from two sources compared to a periodogram of perfect data. Both show periodograms of residuals with only $\gamma$ fit. (a) Actual observational data shown in Figure 7(b) shows the periodogram of a simulated perfect data set for elements shown in Table 14. Panel (b) shows similar features to panel (a), including a peak around 1 day which must be due to sampling.

Table 14

HD 128311: Orbital Parameters and Masses

\begin{tabular}{|c|c|c|c|}
\hline Parameter & HD $128311 b$ & HD $128311 \mathrm{c}$ & HD $128311 d$ \\
\hline \multicolumn{4}{|c|}{ RV } \\
\hline$K\left(\mathrm{~m} \mathrm{~s}^{-1}\right)$ & $55.627 \pm 0.456$ & $74.799 \pm 1.497$ & $13.982 \pm 0.494$ \\
\hline $\operatorname{HET} \gamma\left(\mathrm{m} \mathrm{s}^{-1}\right)$ & $149.989 \pm 0.661$ & & \\
\hline \multicolumn{4}{|c|}{ Astrometry } \\
\hline$\alpha$ (mas) & \multirow{3}{*}{$>30$} & \multirow{2}{*}{$\begin{array}{c}0.456 \pm 0.092 \\
55050+14.553\end{array}$} & \\
\hline$i(\mathrm{deg})$ & & & \\
\hline$\Omega(\operatorname{deg})$ & & $\begin{array}{r}53.930 \pm 14.535 \\
229.392 \pm 14.473\end{array}$ & \\
\hline \multicolumn{4}{|c|}{ Astrometry and RV } \\
\hline$P$ (days) & $453.019 \pm 0.404$ & $921.538 \pm 1.15$ & $11.2210 \pm 0.0008$ \\
\hline$T^{\mathrm{a}}$ (days) & $50198.691 \pm 4.472$ & $49565.615 \pm 18.01$ & $470736.315 \pm 1.112$ \\
\hline$e$ & $0.303 \pm 0.011$ & $0.159 \pm 0.006$ & $0.196 \pm 0.030$ \\
\hline$\omega(\mathrm{deg})$ & $57.864 \pm 3.258$ & $15.445 \pm 6.87$ & $195.545 \pm 8.66$ \\
\hline \multicolumn{4}{|c|}{ Derived $^{\mathrm{b}}$} \\
\hline$a(\mathrm{AU})$ & $1.084 \pm 0.006$ & $1.740 \pm 0.010$ & $0.092 \pm 0.004$ \\
\hline \multirow[t]{2}{*}{ Mass function $\left(M_{\odot}\right)$} & $6.99 \times 10^{-09}$ & $3.84 \times 10^{-08}$ & $2.99 \times 10^{-12}$ \\
\hline & $\pm 1.00 \times 10^{-10}$ & $\pm 1.34 \times 10^{-09}$ & $\pm 1.8 \times 10^{-13}$ \\
\hline$M \sin i\left(M_{J}\right)^{\mathrm{c}, \mathrm{d}}$ & $1.769 \pm 0.023$ & $3.125 \pm 0.069$ & $0.133 \pm 0.005$ \\
\hline$M\left(M_{J}\right)^{\mathrm{d}}$ & & $3.789_{-0.432}^{+0.924}$ & \\
\hline
\end{tabular}

Notes.

${ }^{\text {a }} T=T-2400000.0$.

${ }^{\mathrm{b}}$ An HD 128311 mass of $0.828 \pm 0.012 M_{\odot}$ (Takeda et al. 2007) was used in these calculations.

${ }^{\mathrm{c}}$ The quantity referred to in radial velocity studies as mass, but is actually minimum mass.

${ }^{d}$ Masses are computed from an iteration of

$$
\frac{m 2^{3}}{(m 1+m 2)^{2}}=\frac{a^{3}}{P^{2}} .
$$




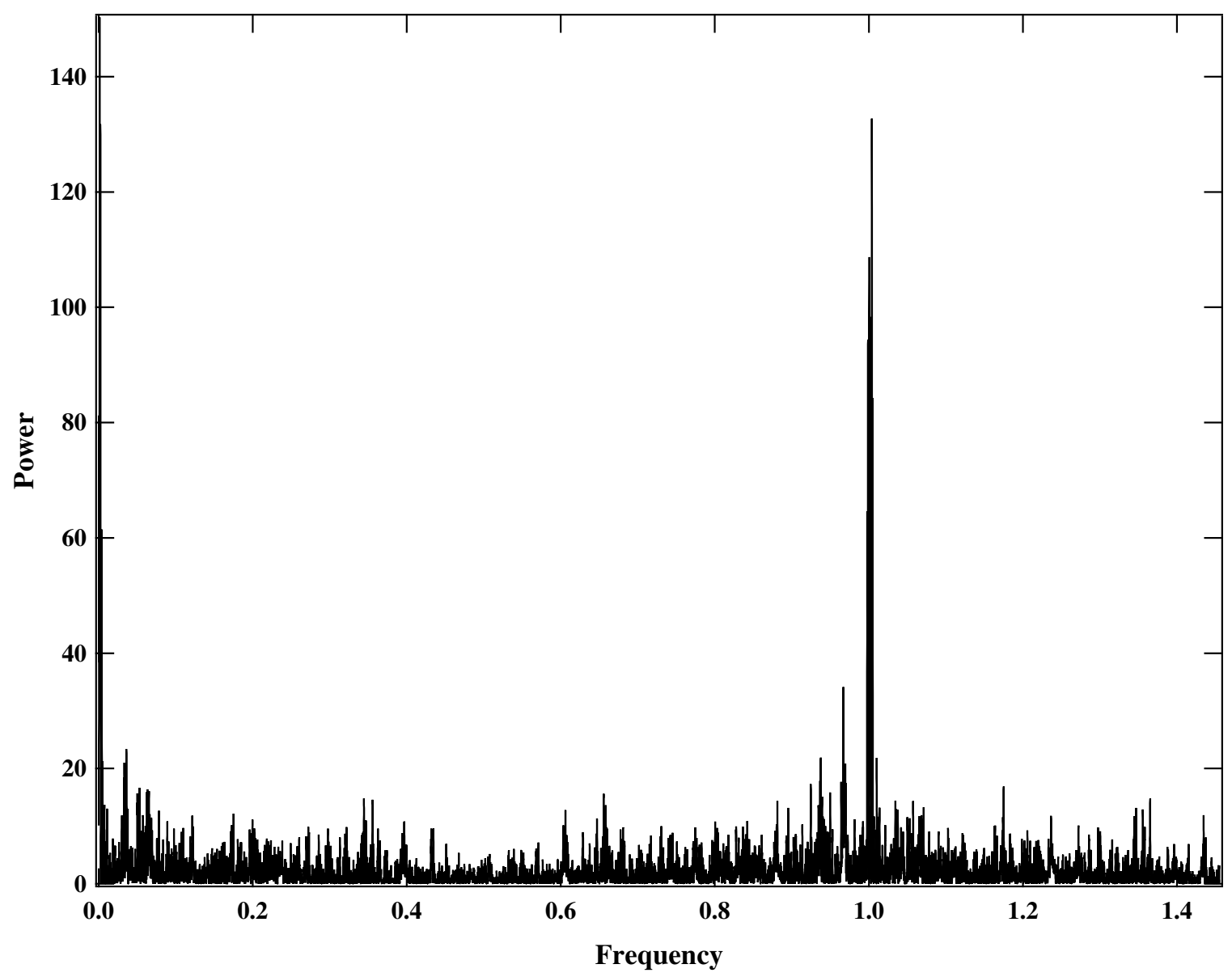

Figure 9. Window function of the radial velocity data of HD 128311 from two sources, showing a peak around one day due to sampling.

in Section 3.4. RV modeling of planets $b-d$ was as detailed in Section 3.5. We constrained the relationship between the astrometry and RV with the equations shown in Section 3.6. We used a tolerance parameter in the GaussFit program that prevents a solution being found in shallow minimas. That tolerance parameter combined with the simultaneous modeling of free parameters (no parameters are held as constants) minimizes the chance of false detection.

Previous investigations (Benedict et al. 2002; McArthur et al. 2004, 2010; Bean et al. 2007; Martioli et al. 2010) and exploratory analysis have shown that we can conservatively detect astrometric signals larger than $\sim 0.25$ mas. Of the two well-determined planets around HD 128311, only planet c would have an astrometric signal that would be detectable by the HST FGS at all inclinations (see Figure 10). Planet b could be detected if its inclination was less than $30^{\circ}$, and planet $\mathrm{d}$ could not be detected at any inclination by HST FGS.

One of the original motivations for these FGS observations was to assess coplanarity of HD $128311 \mathrm{~b}$ and c. Our attempt at the simultaneous modeling of planets $b$ and $c$ failed. With all parameters free, the program iterated endlessly rather than settling on a false detection, indicating that we were not able to detect planet $\mathrm{b}$ astrometrically. However, when we modeled only the astrometric signals of planets $\mathrm{c}$ with the same method, we detected the astrometric signal of planet c. As a test, we attempted to model the astrometric signal of planet $b$ without $c$ and that also failed. The simultaneous modeling of the orbital elements with the astrometry and the RV using GaussFit, with robust estimation, including the astrometric signal of planet $\mathrm{C}$ lowered residuals over the same model which did not include the astrometric signal of planet $\mathrm{c}$.

The orbital elements from this combined simultaneous solution are shown in Table 14 along with the derived elements. The uncorrelated one sigma errors shown are output from the GaussFit program and are produced from a maximum-likelihood analysis of the classic "errors-in-variables" problem. Though not Bayesian, this technique (Jefferys 1990; Jefferys \& Barnes 1999) can be regarded as an approximation to a Bayesian maximum a posteriori estimator with normal priors on certain parameters (see Section 3.4), without the inefficiency and questions of concluding convergence when Markov chain Monte Carlo methods are used.

Using classic astrometric elements to determine orbits does have some degeneracies. When the eccentricity equals zero, $\omega$ (argument of the node) and $T$ (time of periastron) become indeterminate. When $i$ (inclination) is equal to zero (face-on), the $\Omega$ (longitude of the node) becomes indeterminate. Looking at Table 14 , we see that even with $3 \sigma$ errors our determined eccentricities and inclination do not approach zero.

The condition number of a solution indicates the sensitivity of the result to errors (or changes) in the data or model. It gives you and upper bound on the degree to which the errors in the data are magnified in the result. The condition number for this combined model solution is $1.03 \times 10^{+1}$, which means the orbital solution is well constrained, and indicates a loss of only one significant digit in the result from the errors in the data and errors in the model. 


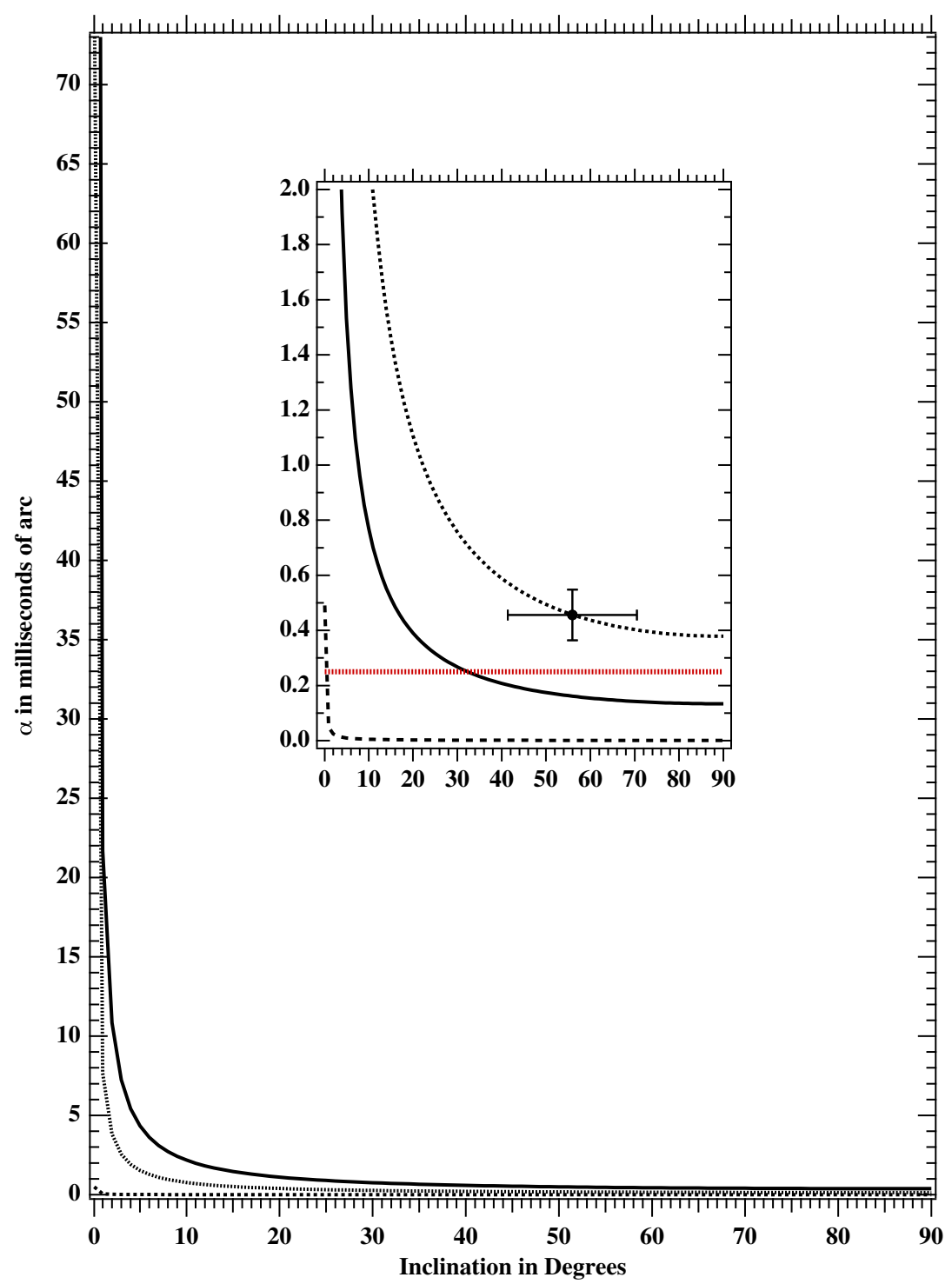

Figure 10. Astrometric $\alpha$, perturbation, against inclination for the three planets in the HD 128311 system as calculated from Equation (8). The HST determined $\alpha$ for HD $128311 \mathrm{c}$ is shown with error bars on the plots. The insert zooms in on components b and $\mathrm{c}$ and shows the detection limit of HST FGS (0.25 mas) in the horizontal dashed line.

(A color version of this figure is available in the online journal.)

The HST astrometric data error estimation is based upon a complex statistical examination of the raw data. We have chosen to continue to use these over-estimated errors because we are not able to assign the overestimation to a particular component of the complex error estimation process. Because of the overestimation of the astrometric data input errors, the actual errors of the astrometric parameters are most likely smaller than listed in the table.

We find the inclination of planet $\mathrm{c}$ to be $55^{\circ} .95 \pm 14.55$. This value is shown in Figure 10 with error bars, illustrating the constraint between astrometric and RV orbital parameters. We place a lower limit on the inclination of HD $128311 \mathrm{~b}$ of $30^{\circ}$ based upon our inability to detect it in our modeling and our confidence in our detection limit. The astrometric motion of planet HD $128311 \mathrm{c}$ against time is shown in Figure 11. The astrometric data shown in these plots (the dark filled circles) are normal points made from the HD 128311 residuals to an astrometric fit of the target and reference frame stars of scale, lateral color, cross-filter, parallax, and proper motion of multiple observations (light open circles) at each epoch.
To calculate companion masses, we use a stellar mass, $M_{\star}$, of $0.828_{-0.01}^{+0.02} M_{\odot}$ for HD 128311, which is a Bayesian-derived determination by Takeda et al. (2007), using the SPOCS catalog. We then find a minimum mass for planet $b$ of $1.77 \pm 0.02$ $M_{\mathrm{JUP}}$ and actual an mass for planet c of $3.789{ }_{-0.432}^{+0.924} M_{\mathrm{JUP}}$. The actual mass was derived from the HST astrometrically measured $\alpha$ 's of planet $\mathrm{c}(0.456 \pm 0.092)$. The minimum mass, $M \sin i$, for the possible planet $\mathrm{d}$ is $0.133 \pm 0.005$ $M_{\mathrm{JUP}}$. For possible planet d using Equation (1) of Bodenheimer et al. (2003), we find the probability of transit to be from $4 \%-5 \%$ dependent upon assumptions about planet d's radius and temperature.

The $\gamma$ adjusted velocities from the HET and Lick observatories with the combined orbital fit of HD $128311 \mathrm{~b}$, c and d over plotted are shown in the top panel of Figure 12 and the velocity residuals are shown in the lower panel. Table 15 shows the number of observations and rms of the two RV data sources with a weighted average rms of $9.37 \mathrm{~m} \mathrm{~s}^{-1}$. The rms of the HET data was $8.37 \mathrm{~m} \mathrm{~s}^{-1}$. Figure 13 shows the RV of companions $\mathrm{b}-\mathrm{d}$ (with the other component velocities removed) plotted 

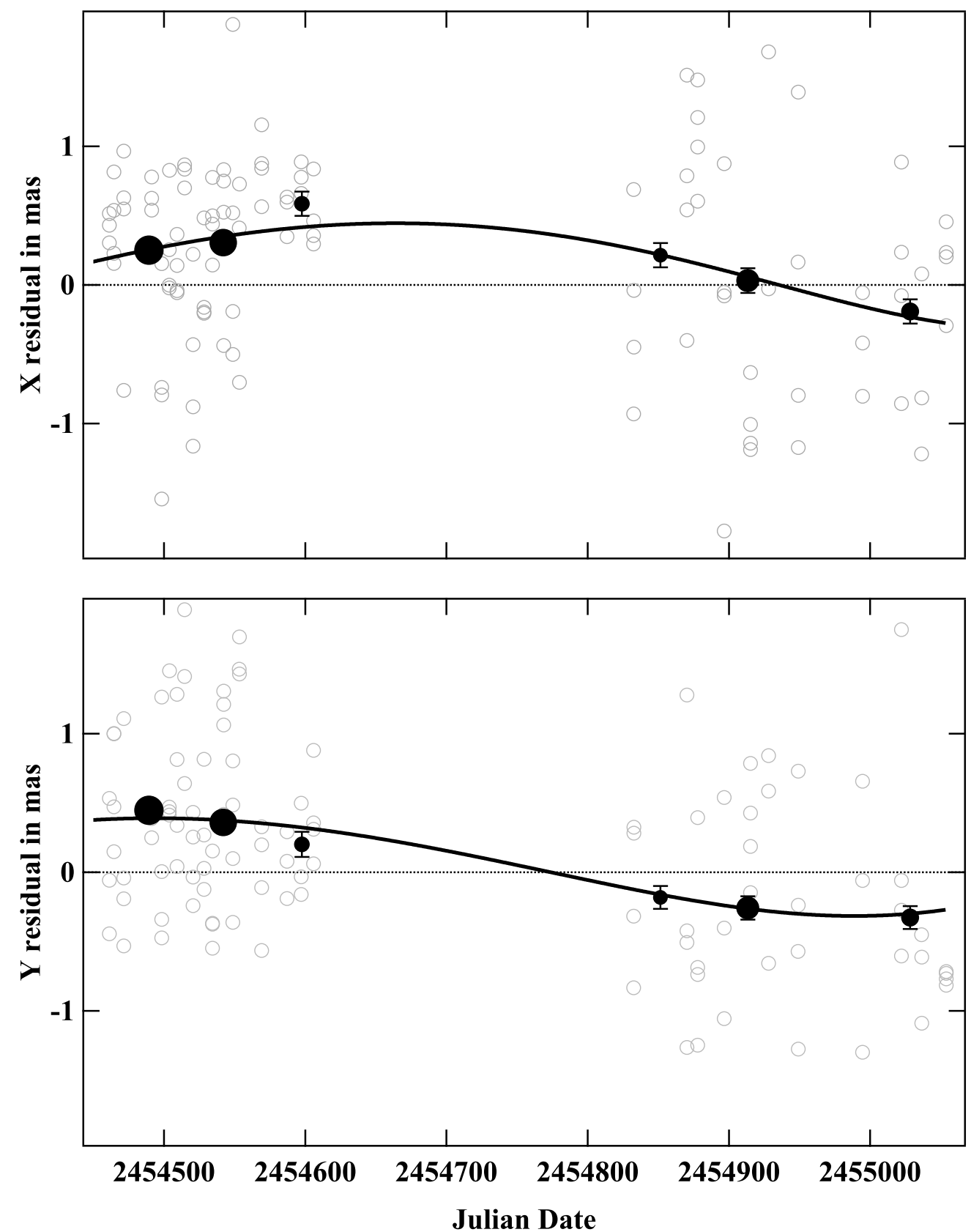

Figure 11. Astrometric reflex motion of HD 128311 due to c against time is shown. The astrometric orbit is shown by the dark line. Dark filled circles are normal points made from the HD 128311 residuals to an astrometric fit of the target and reference frame stars of scale, lateral color, cross-filter, parallax, and proper motion of multiple observations (light open circles) at each epoch. Normal point size is proportional to the number of individual measurements that formed the normal point. The peak-to-peak separation between the normal points, which are near peri- and apastron, is two times the perturbation semi-major axis $(2 \alpha)$, by definition. Error bars represent the $1 \sigma$ of the normal position. Many error bars are smaller than the symbols.

Table 15

Radial Velocity Data Sets of HD 128311

\begin{tabular}{lccr}
\hline \hline Data Set & Coverage & Number of Observations & $\begin{array}{c}\mathrm{rms} \\
\left(\mathrm{m} \mathrm{s}^{-1}\right)\end{array}$ \\
\hline Lick & 1998 June-2008 May & 92 & 13.21 \\
McDonald HET & 2005 Apr-2011 Jan & 355 & 8.37 \\
\hline Total & & 974 & 9.37
\end{tabular}

against orbital phase. The histogram in Figure 14 shows the Gaussian distribution of the HET RV residuals of the combined orbital model which include residuals from two different sources spanning $13 \mathrm{yr}$.

\subsubsection{Proper Motion and Orbital Parameters in Astrometry}

Black \& Scargle (1982) suggested that the linear component of an astrometric perturbation $(\alpha)$ is absorbed into the proper motion and therefore both the period and amplitude of the orbital motion are underestimated unless several orbital cycles are observed. However, their hypothesis considers the astrometric orbit as being sinusoidal (circular, ecc $=0$ ) and linear with time (see Figure 3 of Black \& Scargle 1982). An eccentric orbit is not sinusoidal. A two-axis astrometric orbit with eccentricity is never linear in time. To test the Black and Scargle hypothesis, an "almost-perfect" data set was created with orbit, proper motion and observing times to match the 


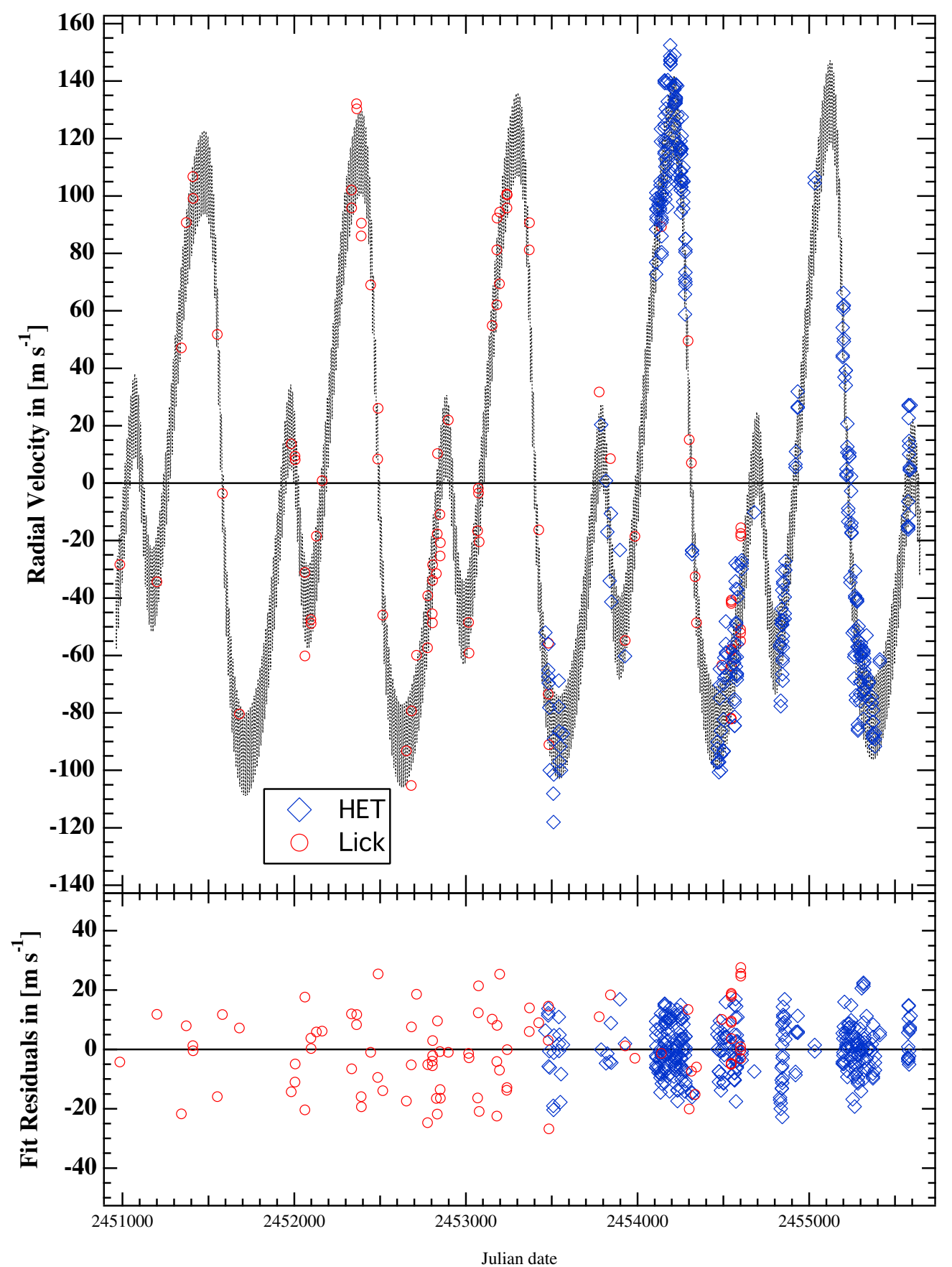

Figure 12. Radial velocities of HD 128311 vs. time from two sources. The orbital parameters were established with a simultaneous three-planet Keplerian orbit to all Doppler measurements combined with HST astrometry. The solid gray line shows the combined orbital fit which includes planets b, c, and d. The lower panel shows the residuals to the fit.

(A color version of this figure is available in the online journal.)

HST astrometry of HD 128311c. Without including the RV, and solving for all astrometric parameters simultaneously, we used our GaussFit model to determine the proper motion and orbital parameters, and compared the results with the input parameters to the fake data set of the proper motions and astrometric $\alpha$. The $\mu_{\alpha}$ difference was $-3.377 \times 10^{-6}, \mu_{\delta}$ difference was $1.532 \times 10^{-6}$, and the astrometric $\alpha$ difference was $1.309 \times 10^{-6}$, which are at the level of error in the fake data set. With $64 \%$ orbital coverage, we are able to recover the input parameters, without transfer of orbital motion into proper motion.
With accurate astrometric data, it is possible to determine orbital parameters with an arc of the orbit. It is only necessary to have enough astrometric data to be able to separate the nonlinearities of the orbital motion from the linear proper motion. Combining RV data of more than one period in a simultaneous model with the astrometry allows determination of astrometric parameters with observations times that stretch only one-tenth of the orbital period (Tuomi et al. 2009). The inclusion of the RV observations would also prevent absorption of the astrometric perturbation in a zero eccentricity orbit into the proper motions because the RV 


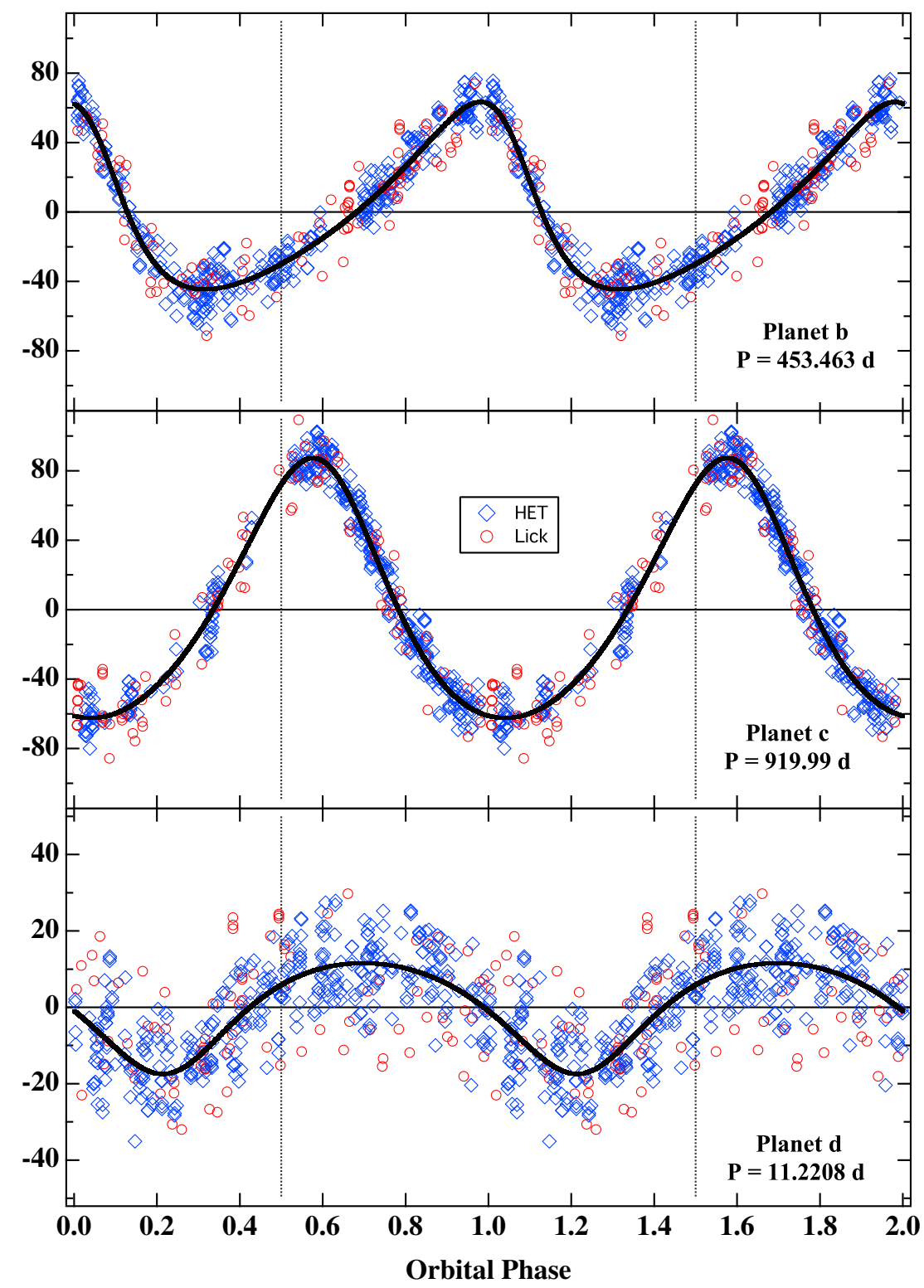

Figure 13. Residual velocities of HD 128311 vs. orbital phase for each planet after the subtraction of the signal produced by the two other planets. The orbital parameters were established with a simultaneous three-planet Keplerian fit to all Doppler measurements combined with HST astrometry. The solid line shows the Keplerian curve of the planet alone.

(A color version of this figure is available in the online journal.)

would constrain any period change that covaries with the $\alpha$ absorption.

\subsubsection{Hipparcos versus HST Observations}

Hipparcos astrometry measurements are inherently onedimensional. HST astrometric measurements are two-axis interferometric. Several studies have shown that Hipparcos intermediate astronomic data (IAD) measurements do not have enough precision to determine planetary orbit, but can be used to set upper limits on planet size (Sozzetti 2005). However, with the rereduction of the Hipparcos catalog in 2007 (van Leeuwen 2007), masses have been derived for substellar companions (Sozzetti \& Desidera 2010; Reffert \& Quirrenbach 2011). The technique used for these mass determinations uses the RV (spectroscopic) elements as constants and only solves for the astrometric $i$ (inclination) and $\Omega$ (ascending node). The $\alpha$ (semi-major axis) is derived from a constraint with the spectroscopic elements held as constants (see Sozzetti \& Desidera 2010, Equation (1)).
Because their semi-major axis is derived, Sozzetti \& Desidera (2010) use Monte Carlo resamples to estimate the $\alpha$ error. The $\alpha$ is actually measured with the HST FGS observations in a simultaneous solution of all spectroscopic and astrometric elements, with the error directly calculated, as with the other parameters in the GaussFit model solution. Reffert \& Quirrenbach (2011) did confirm the HST FGS results for $\epsilon$ Eri b (Benedict et al. 2006).

\subsubsection{Modeling of the HST Astrometric Residuals}

An noted in Section 4.2.2, the HST astrometric data is modeled for scale, lateral color, cross-filter, proper motion, and parallax. As an additional test of the astrometric signal, we model the astrometric data with the above parameters, but without the orbit of planet $c$ and examine the residuals of that fit. We modeled the astrometric residuals (with reduced input errors) with a straight line fit in each axis over time, and with an orbit over time. The reduced $\chi^{2}$ of the linear fit model was 


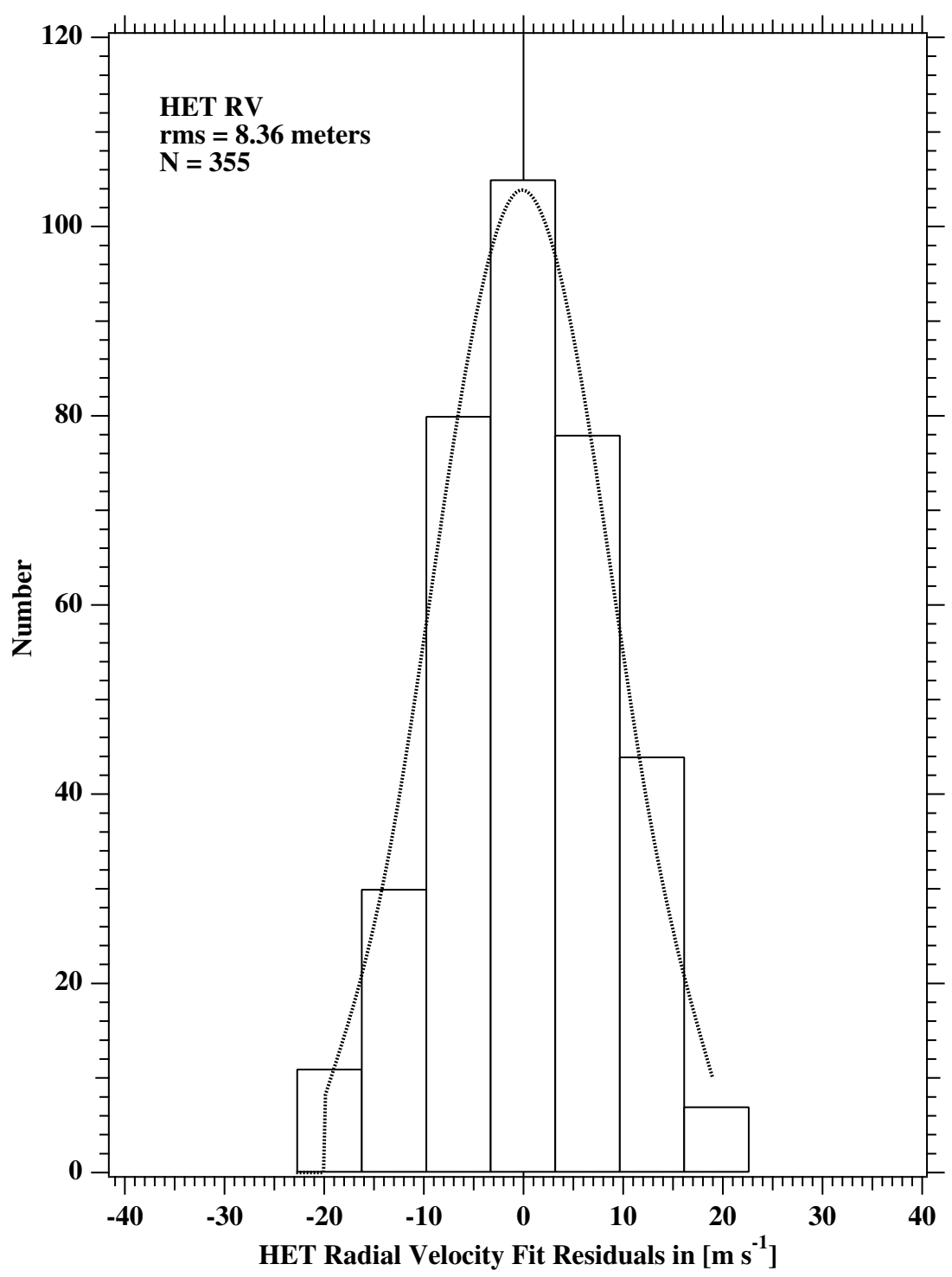

Figure 14. Histogram of the HET RV residuals of the simultaneous three-planet Keplerian Orbit fit to all Doppler measurements combined with HST astrometry of HD 128311.

14.17, while the reduced $\chi^{2}$ of the orbital model was 3.00. The astrometric residual fit to an orbit was almost five times lower than the fit to a straight line, which supports the determination of an orbital signal in the residuals.

\subsection{Photometry of HD 128311}

In addition to our RV and astrometric observations described above, we acquired contemporaneous photometric observations of HD 128311 with the T12 0.80 m APT at Fairborn Observatory in southern Arizona. T12 is one of several Tennessee State University automatic telescopes in operation at Fairborn (Eaton et al. 2003). We also analyzed our astrometric observations with the HST FGS 1r, a millimag photometer (Benedict et al. 1998; Bean et al. 2008), to produce additional photometric observations simultaneous to the astrometric observations.

Precise photometric observations of planetary host stars are useful to look for short-term, low-amplitude brightness variability that may be the result of rotational modulation in the visibility of starspots and plages (see, e.g., Henry et al. 1995). Therefore, these observations can help to determine whether observed RV variations are caused by stellar activity or reflex motion due to the presence of an orbiting companion. Queloz et al. (2001) and Paulson et al. (2004) have documented several examples of solar-type stars whose periodic RV variations were caused by stellar activity. Photometric observations of HD 128311 are of particular importance to establish the reality of the new 11.22 day planetary candidate HD $128311 \mathrm{~d}$ seen in our RVs, given that Vogt et al. (2005) have suggested (from examination of $\mathrm{Ca}$ II $\mathrm{H}$ and $\mathrm{K}$ lines) that this active star has a rotation period of $\sim 12$ days.

The T12 APT acquired 375 observations of HD 128311 during the 2004, 2008, 2009, and 2010 observing seasons. The T12 APT is equipped with a two-channel precision photometer employing two EMI 9124QB bi-alkali photomultiplier tubes to make simultaneous measurements in the Strömgren $b$ and $y$ passbands. The T12 APT and its two-channel photometer are nearly identical to the T8 APT described in Henry (1999). The APT was programmed to measure the brightness of HD 128311 (star $D: V=7.48, B-V=0.97, \mathrm{~K} 0$ ) with respect to the three nearby comparison stars HD 129153 (star $A: V=5.93$, $B-V=0.24$, F0 V), HD $125752(\operatorname{star} B: V=7.97$, $B-V=0.40, \mathrm{F5})$, and HD 127247 (star $C: V=7.41$, 


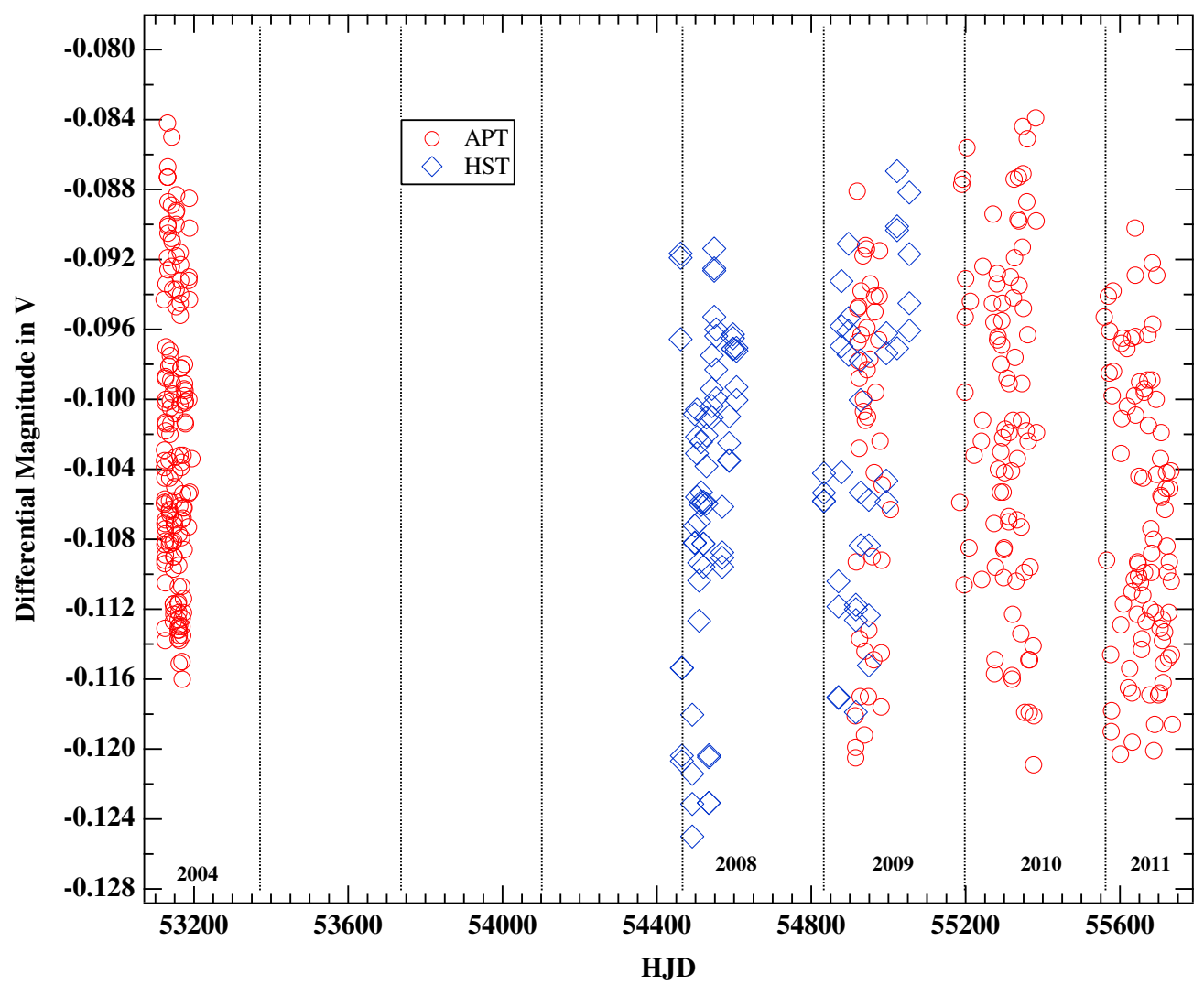

Figure 15. Photometry from both the HST and T12 APT of HD 128311 against time in HJD. T12 APT are differential magnitudes and the HST FGS data have been offset to appear on the plot.

(A color version of this figure is available in the online journal.)

Table 16

HST Photometry of HD 128311

\begin{tabular}{lc}
\hline \hline Julian Date & Magnitude \\
\hline 2454461.32336805 & 7.3987 \\
2454461.33312499 & 7.3937 \\
2454461.33790509 & 7.3984 \\
2454464.51170138 & 7.3749 \\
2454464.52334490 & 7.3699 \\
\hline
\end{tabular}

(This table is available in its entirety in a machinereadable form in the online journal. A portion is shown here for guidance regarding its form and content.)
Table 17

T12 APT Photometry of HD 128311

\begin{tabular}{lc}
\hline \hline Julian Date & Differential Magnitude \\
\hline 2453122.6862 & -0.0943 \\
2453123.7842 & -0.106 \\
2453123.8253 & -0.1059 \\
2453123.8326 & -0.1029 \\
2453123.8829 & -0.1035 \\
\hline
\end{tabular}

(This table is available in its entirety in a machinereadable form in the online journal. A portion is shown here for guidance regarding its form and content.)
$B-V=0.53, \mathrm{~F} 2)$. Intercomparison of the various differential magnitudes of the four stars shows that comp star $A$ is slightly variable over a range of a few millimagnitudes, comp stars $B$ and $C$ are constant to $0.001 \mathrm{mag}$ or so, and our program star HD 128311 ( $\operatorname{star} D$ ) is variable over a range of $\sim 0.03$ mag. Therefore, we created the most precise differential magnitudes of HD 128311 by averaging the $D-B$ and $D-C$ differential magnitudes into a single $D-(B+C) / 2$ differential magnitude. We also combined our $b$ and $y$ observations into a single $(b+y) / 2$ passband to improve the precision further. Finally, since the mean brightness of the four observing seasons varied slightly from year to year with a standard deviation of 0.0028 mag, we normalized each observing season to the same mean and computed the individual residuals from the mean. The four observing seasons of the APT data are plotted in Figure 17 with filled circles.
We combined the 108 HST FGS measurements listed in Table 16 spanning 1.62 yr with 375 additional observations from the T12 APT shown in Table 17 spanning (with gaps) $7.15 \mathrm{yr}$ for the photometric analysis (see Figure 15). Typically the HST target star photometry is flat-fielded with the stars from its reference frame, but in the case of HD 128311 the only two reference stars that were in all of the observation sets were very faint reference stars with low pmt counts. The attempt to flat-field the data with these stars resulted in considerable noise being added to the data. Because we were unable to flat-field the data the HST photometry is expected to be of lower quality than usually produced. The HST magnitudes were derived from a relation between the sum of pmt counts of four channels with SIMBAD magnitudes of non-variable HST observed stars.

The Lomb-Scargle periodograms shown in Figure 16 of the HST photometry show a period of 11.1796 days with a probability of false detection of $1.223571 \times 10^{-10}$ and T12 


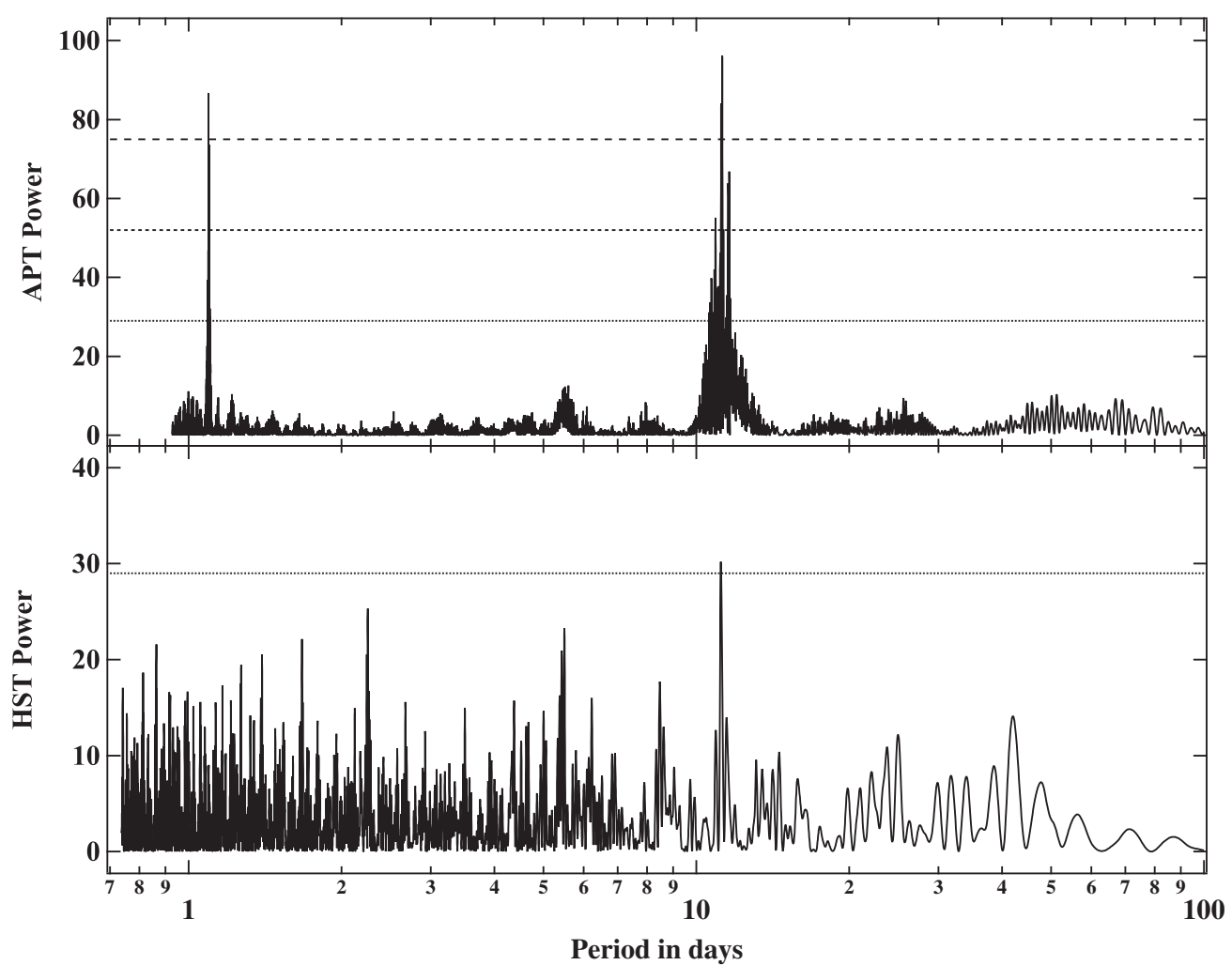

Figure 16. Periodogram of the photometry data of HD 128311 from two sources. Upper plot shows T12 APT photometry with a maximum peak at 11.248625 days and a false alarm probability of $9.854567 \times 10^{-39}$. The dotted lines show the significance levels of the power, from the top: $1.0 \times 10^{-30}, 1.0 \times 10^{-20}, 1.0 \times 10^{-10}$. Lower plots show HST photometry with a maximum peak at 11.179618 days and a false alarm probability of $1.223571 \times 10^{-10}$, only the significance level line of $1.0 \times 10^{-10}$ is shown.

Table 18

Photometric Period Modeling of HD 128311

\begin{tabular}{lllclrr}
\hline \hline ID & \multicolumn{1}{c}{ Date } & \multicolumn{1}{c}{ Period } & Amplitude & rms & $\chi^{2}$ & DOF \\
\hline T12 APT & Apr 4-Jul 4 & $11.33340 \pm 0.03667$ & $0.009 \pm 0.001$ & 0.00435 & 149 & 149 \\
T12 APT & Mar 9-Jun 9 & $11.25298 \pm 0.00099$ & $0.012 \pm 0.001$ & 0.00478 & 53 & 41 \\
T12 APT & Dec 9-Jul 10 & $11.25588 \pm 0.00060$ & $0.011 \pm 0.001$ & 0.0044 & 88 & 83 \\
T12 APT & Dec 10-Jun 11 & $11.24745 \pm 0.00055$ & $0.009 \pm 0.001$ & 0.0041 & 79 & 86 \\
$H S T$ & Dec 7-May 8 & $11.25000 \pm 0.0000006$ & $0.0117 \pm 0.001$ & 0.0069 & 44 & 64 \\
$H S T$ & Jan 7-Aug 9 & $11.24999 \pm 0.0000009$ & $0.0101 \pm 0.001$ & 0.0069 & 19 & 37 \\
\hline Combined & Apr 4-Jun 11 & $11.25018 \pm 0.0008$ & $0.009 \pm 0.0004$ & 0.00054 & 583 & 468 \\
Combined & Dec 7-Jun 11 & $11.25152 \pm 0.0018$ & $0.009 \pm 0.0005$ & 0.00054 & 466 & 317 \\
\hline
\end{tabular}

APT photometric data showed a period of 11.2486 days with a probability of false detection of $9.854567 \times 10^{-39}$. GaussFit was used for simple phase modeling of the photometric data from both instruments using this equation:

$$
\Phi=a+b * \Delta t+A * \sin \left(\frac{2 * \pi * \Delta t}{P}\right),
$$

where $a$ is an offset and $b$ is a linear trend term for each season of data (four for T12 APT and one for HST), $\Delta t$ is the change in time, $A$ is the amplitude of the sine wave, and $P$ is the period in days of the photometry.

While there is an indication from comparing a polynomial fit to the sine fit of the photometric data that there are subtle low level differences, the sine wave photometric model appears to be adequate for the period determination. Adding an eccentric component to the sine fit did not improve the results. Table 18 lists the period of each season separately and simultaneously, along with the rms of the fit and DOF and $\chi^{2}$. The 2004 T12
APT data is much noisier in regard to the period determination as can be seen from the table. We did allow for a single time offset in the Julian Dates of the observations from the two instruments.

Figure 17 shows the coherent photometric signal in sequential time. It is interesting that the modeled period derived from HST photometry is significantly different than the result from the periodogram and closer to that derived from the T12 APT data. The photometric period found in the modeling of all epochs of both data sets is $P_{\text {phot }}=11.25145 \pm 0.00046$ days. For the simultaneous modeling of both sets of data the variance $\left(\sigma^{2}\right)$ used for the T12 APT data was $2.6 \times 10^{-5}$, while the variance used for the HST data was $1.95 \times 10^{5}$. In the top panel of Figure 18 we see both sources of photometric data phased to this period. The lower panel shows all photometric data phased to the period of the $d$ component RV variations ( $P_{\mathrm{RV}}=11.2210$ days $)$. While the periods of the photometry and the RV are very similar, they are not the same within the errors. 


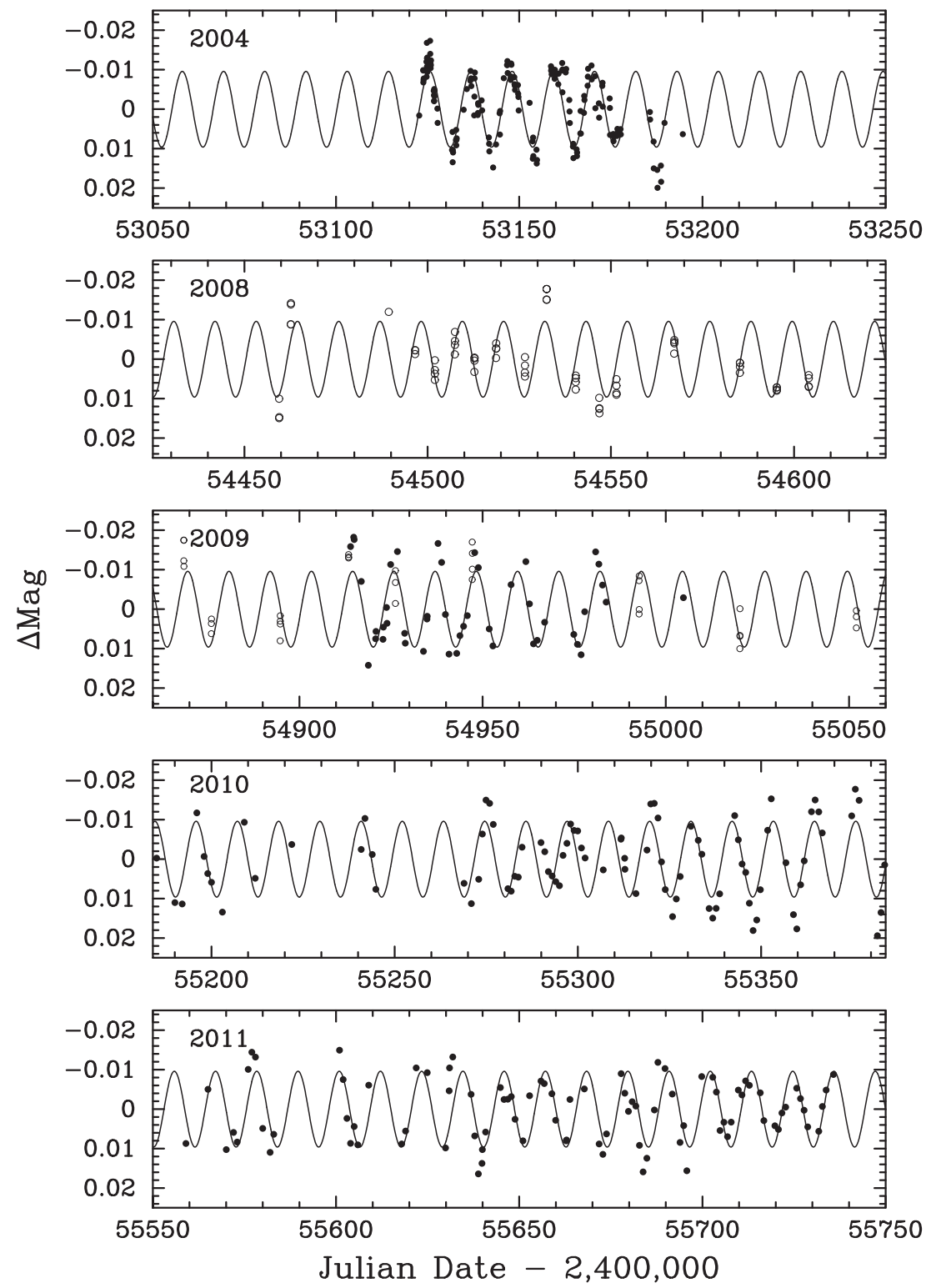

Figure 17. Plot of the photometry data of HD 128311 from two sources against time by year showing coherency over 8 yr. The peak-to-peak amplitude of the fitted sine curve is $0.01920 \pm 0.00066 \mathrm{mag}$. The filled circles in the plot are APT data. The open circles are the HST data.

\subsection{Cross-correlation Bisector Analysis}

In order to assess the origin of short period RV variations we performed a bisector analysis on data from the HET. We found that on one of the two observing tracks of the HET close to twilight there were very large outliers in bisector span measurements as shown in Figure 19. The magnitude of the bisector span outliers was much larger than the uncertainty attributed to these observations in the $\mathrm{RV}$ reduction process. We removed these western track, near twilight observations from our RV and bisector data sets.

Because of the similar photometric and RV periods ( $P_{\text {phot }}$, $P_{\mathrm{RV}}$ ), we examined the bisector of the cross-correlation function (CCF) of 274 HET RV observations to look for correlations with the 11.2210 day RV period. We look for distortions in the CCF bisector that occur because of the movement of starspots across the limbs of the star as it rotates. We used the method of Johns-Krull (see Mahmud et al. 2011; Huerta et al. 2008 , for recent discussions). The bisector spans and offsets were determined by cross correlating 12 orders covering the wavelength range 4387-4847 $\AA$. The values represent the mean CCF bisector span computed from the CCF of the 12 orders and the error is the standard deviation in the mean of the 12 orders. We found no correlation between the CCF bisector spans and the RV residuals (Figure 20). We did find a correlation between the bisector span uncertainties and the day of the year and track of the observations at the HET and used these to filter the higher noise HET data as explained in Section 3.1.

\subsection{Dynamical Stability Analysis}

Early dynamic work (Vogt et al. 2005) suggested that the system parameters derived from the Lick RV measurements most certainly required resonance for stability. Our new analysis uses Mercury (Chambers 1999) with a wrapper STAB (by Martioli) that provides a method for automatically inputting orbital elements with uncertainties that are incremented by either a Gaussian or uniform distribution. Unfortunately, Mercury does not implement the effects of general relativity, which could affect the stability of the system. Therefore, we can expect that 


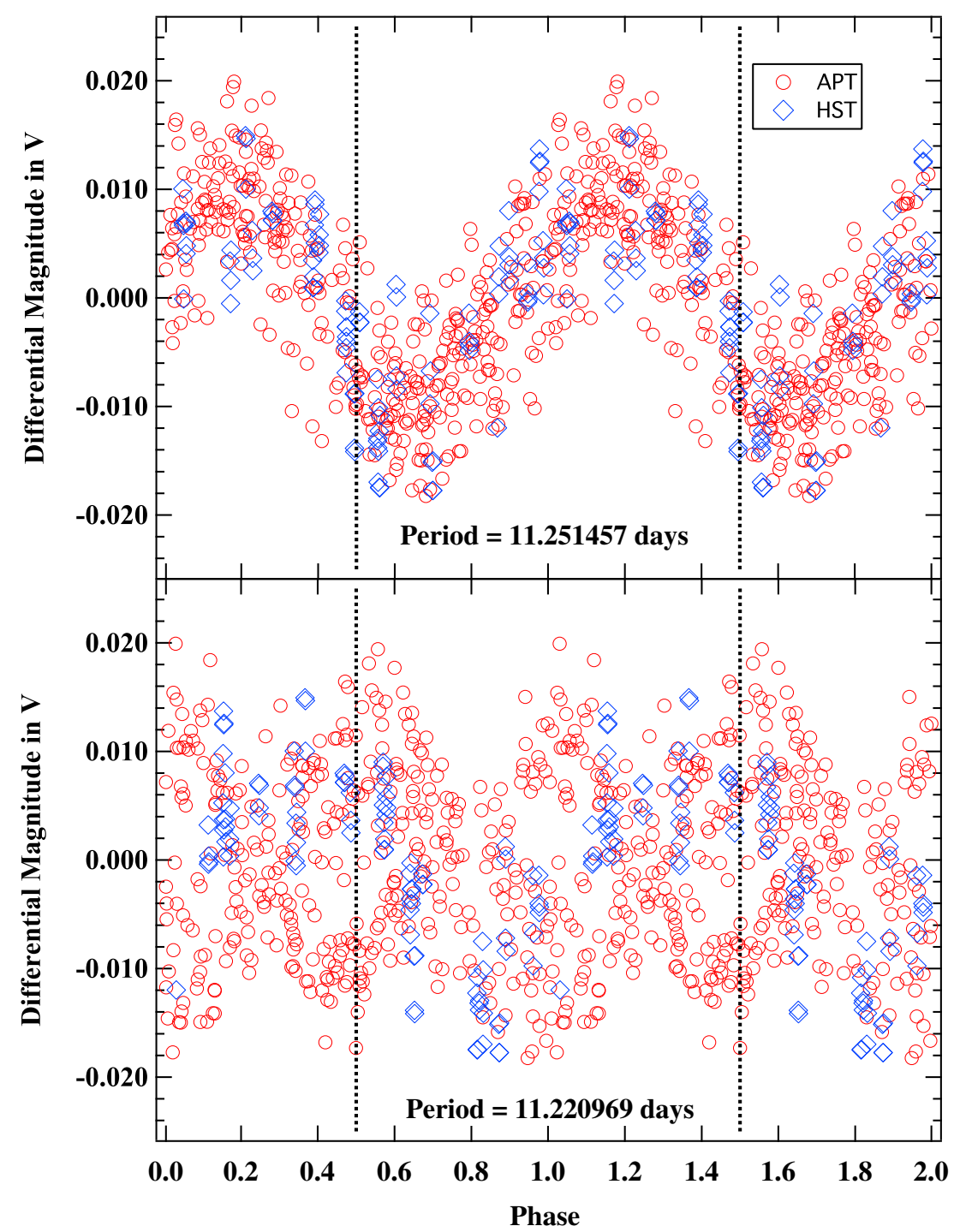

Figure 18. Phase plot of the photometry data of HD 128311 from two sources. Upper plot shows T12 APT and HST photometry phased to the photometry modeled period of 11.25145 days. The lower plots show T12 APT and HST photometry phase to the radial velocity signal of 11.220969 days.

(A color version of this figure is available in the online journal.)

these initial results may present a more minimal picture of the regions of stability in the dynamical map.

In this preliminary investigation, we used the astrometric and RV elements and errors in Table 14 for HD 128311 for planets b and $\mathrm{c}$ as inputs into STAB (STAB converts the orbital elements into canonical coordinates). We use the Bullrisch-Storer integration, with time steps of 22 days, and a Julian day start time of 2453462.0 and stop time of 3654953462.00 days. We found stability for the system for 10 million years.

To examine the claim of resonance in the HD 128311 system we looked at the $\psi$ 's calculated with this equation:

$$
\psi=p * M_{1}-q * M_{2}-(p-q) * \varpi_{1 o r 2},
$$

where $p=2$, and $q=1$ for this system, $M$ is the mean longitude, and $\varpi$ is the longitude of pericenter. We see in Figure 21 that the resonant angles are circulating, which indicates that there is no resonance. If the system were in resonance, all the data would be confined to a certain range on the $y$-axis (usually, but not always $0^{\circ}$ or $180^{\circ}$ ).

\section{DISCUSSION}

\subsection{Possible Coplanar Architecture}

The astrometric data set for the determination of orbital parameters of HD 128311 was less robust than our past data sets for complex multi-planet systems. We had only 29 orbits in contrast to the 54 orbits we had for $v$ Andromedae, and these orbits were interrupted by several months for observing constraints, producing a data gap. The measurements were spread out over time with none of the intense monitoring that we had for $v$ Andromedae. We were only able to observe at maximum parallax factors twice due to $H S T$ instrumental restrictions on telescope roll, we had a large 225 day gap in the middle of our observation set, and our reference frame was very faint, with data losses on different stars in different orbits. All of these things combined produced a less than optimum data set. Nevertheless, we did find an inclination of planet c of $55.95 \pm 14.55$ and were able to place a lower limit on the inclination of HD $128311 \mathrm{c}$ of $30^{\circ}$. These measurements support the possibility of coplanar architecture for HD $128311 \mathrm{~b}$ and c. 


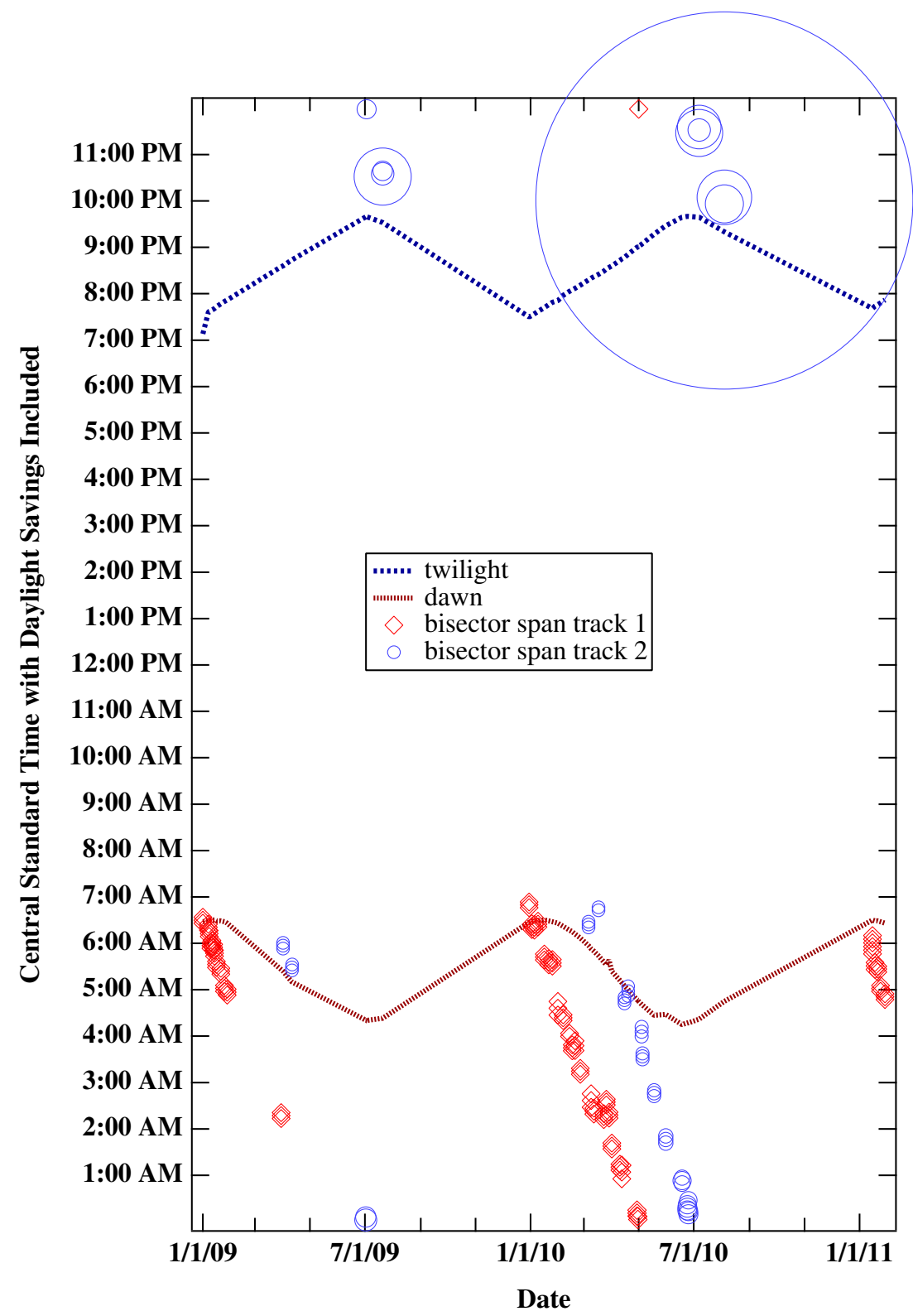

Figure 19. Results of bisector span analysis of HET radial velocity data of HD 128311 plotted against date and time. Time is shown as central standard time with daylight saving included. Twilight and dawn are indicated. The HET has two tracks-track $1(\diamond)$ when azimuth is approximately $110^{\circ}(\mathrm{E})$ and track $2(\bigcirc)$ with an azimuth of approximately $240^{\circ} \mathrm{W}$ ). Symbol sizes indicate bisector span size. Large outliers exist near twilight on track 2 (W). These outliers (large circles at the top of the figure) were removed from the HET radial velocity data set.

(A color version of this figure is available in the online journal.)

\subsection{Detection of an Additional Planet or Activity Cycle?}

In active stars, rotational modulation of surface phenomenon or pulsations can mimic RV variations that are due to reflex motions induced by planetary companions. The signature of rotational modulations are usually sinusoidal and varying over time (because of the growth and decay of the individual starspots) and are identified by periodogram analysis of the measured $S$ (Ca II $\mathrm{H}$ and $\mathrm{K}$ index) values (although there can be phase shifts between the $S$ value variation and the RV variation). We were not able to compute a Mount Wilson " $S$ " index because our HET HRS spectrum do not cover the wavelength region of the $\mathrm{Ca}$ II $\mathrm{H}$ and $\mathrm{K}$ lines.

$\mathrm{RV}$ and photometric variations with periods of a few days due to pulsations have been detected in K-type stars, but these are all giant stars (Hatzes \& Cochran 1993, 1994). Stellar pulsations with periods of a few days in a K dwarf, which HD 128311 is, would be unprecedented and there is only one (USNW-V-760) unconfirmed report of possible pulsations in such a star (Koen 2011, 2012). From a theoretical study of the radial pulsations of low mass stars (Gabriel \& Grossman 1977), the pulsation period of the K dwarf would be expected to be much shorter than the observed photometric variation $\left(P_{\text {phot }}=11.25145\right)$ we see in HD 128311.

Because of concerns about planet detection around active stars, Butler et al. (2003) evaluated Mount Wilson Ca II H and K $S$-values of HD 128311 from their Keck spectra and only found two periods at 31 and 25 days that were close to the $1 \%$ false alarm probability. They also suggested that monitoring of the system would show excursions from the Keplerian 


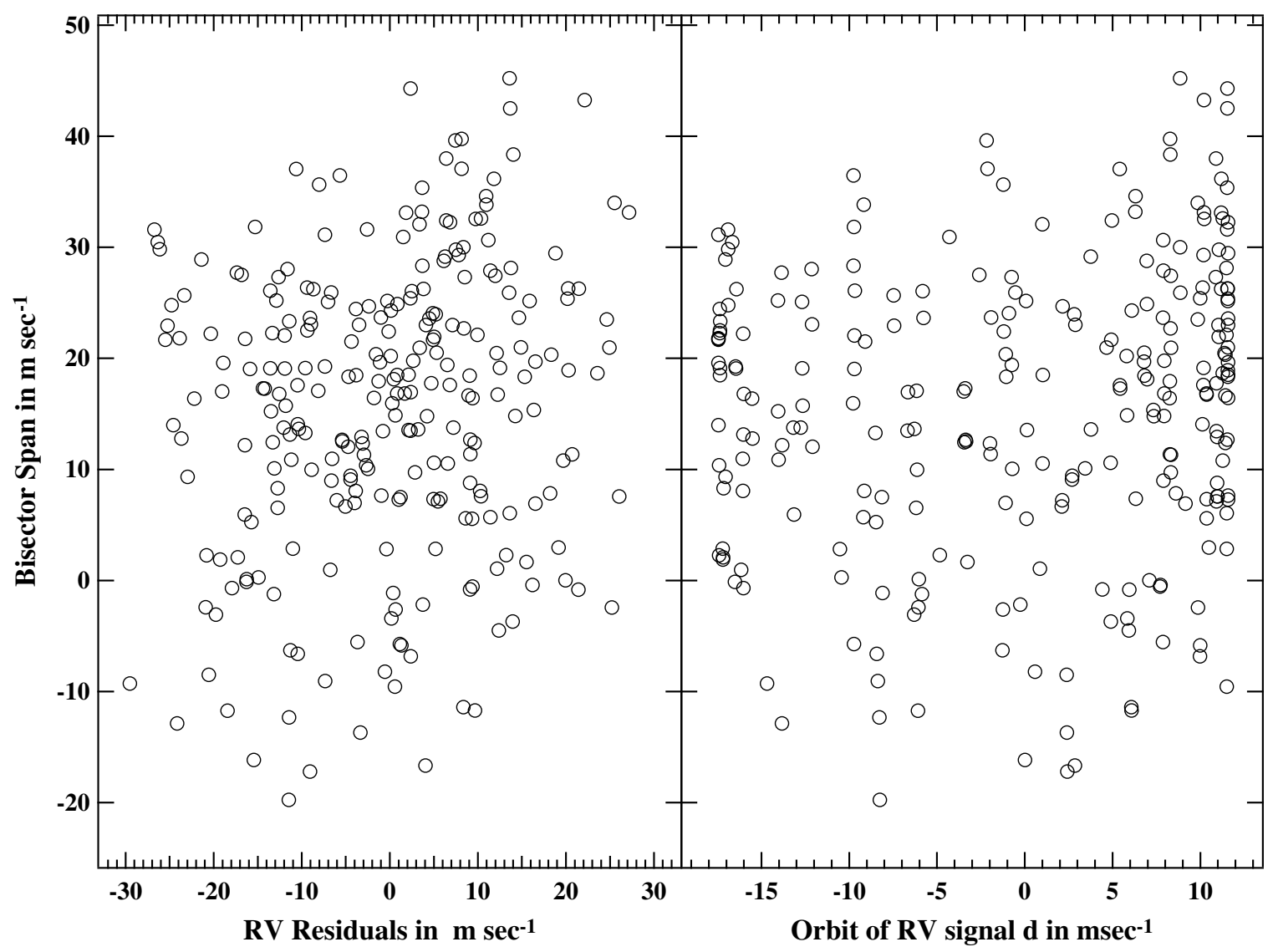

Figure 20. Plot of the bisector spans and radial velocity fit residuals of HD 128311 and a perfect orbit. A correlation between the bisector spans and the radial velocity residual would indicate star activity as the source of the radial velocity variations. Left panel shows the bisector spans against the radial velocity residual after the fit of components $\mathrm{b}$ and $\mathrm{c}$. Right panel shows the bisector span against the modeled radial velocity signal of possible companion d, to minimize the noise of the actual observations. Lack of correlation suggests a planetary origin.

orbit, if the RV variation were being caused by other star phenomenon.

In the followup announcement of the discovery of the second planetary body in the system, Vogt et al. (2005) found strong evidence of a RV periodicity at 5.6 days and speculated that this period was caused by spots on opposite hemispheres of the stars rotating across the disk. Strassmeier et al. (2000) had first identified a photometric period 11.5 days with an amplitude of 0.035 mag. Vogt et al. (2005) later found a period of $11.53 \pm$ 0.15 days with 0.03 mag amplitude in photometric variability. They predicted the rotation period to be $P_{\text {rot }}=12$ days from emission at $\mathrm{Ca}$ II $\mathrm{H}$ and $\mathrm{K}$, which they found it to be in agreement with the photometric period of 11.53 days, which also explained to them the 5.6 day RV period.

As discussed in Section 4.2.1, after removal of the two known planets $\mathrm{b}$ and $\mathrm{c}$, a strong RV signal remains at $\mathbf{1 1 . 2 2}$ days which can be seen in Figure 7. This period is twice that of the 5.6 day period found by Vogt et al. (2005) in the residuals to their two planet fit. We do not see the shorter 5.6 signal in our periodogram (see Figure 7) or in a plot of residuals to a two planet fit phased to 5.6 days. The HET data set has much higher cadence over a significant period of time compared to the Lick data (see Figure 12). We found a significant drop of over $50 \%$ in the $\chi^{2}$ of the solution with the addition of the Keplerian orbit planetary argument $\mathrm{c}$ to the model, shown in Table 13.

Because the origin of the RV variations was not related with certainty to the photometric variability we turned to additional methods of discernment. Bisectors of spectral lines (Gray 1976; Hatzes et al. 1997) or the CCF's can be examined to rule out rotational modulations of starspots and non-radial pulsations. If there is asymmetry in the CCFs that is correlated with the $\mathrm{RV}$ variations, then a planetary companion can be ruled out. Without correlation, radial pulsations or a planetary companion can still be considered. In the case of HD 128311 radial pulsations are unlikely at the period of the RV signals. In the past, after further examination, tentative planets in HD 166435 (3.7987 days; Queloz et al. 2001) and more recently BD +20 1790 (Hernán-Obispo et al. 2010; Figueira et al. 2010) were ruled out when the RV variations proved to be due to stellar activity. Chromatic variations that result in RV amplitude that is wavelength dependent can also be indicators that the signal is caused by rotational modulation or pulsations.

We proceeded with additional examinations of the photometry (see Section 4.3) and bisectors (see Section 4.4) to determine the origin of the residual periodic RV observations. T12 APT and HST photometry showed a robust photometric period of $P_{\text {phot }}=11.25145 \pm 0.00046$ days which is very close, but not within the errors to RV period of $P_{\mathrm{RV}}=11.2210 \pm 0.0008$. We see the that the photometry is much more compelling phased to the period determined by it rather than by the RV $P_{\mathrm{RV}}$ (see Figure 18).

If HD 128311 is a low amplitude starspot variable, the coherency of the time period of the photometric observations is unusual. Starspots in these types of stars typically appear 


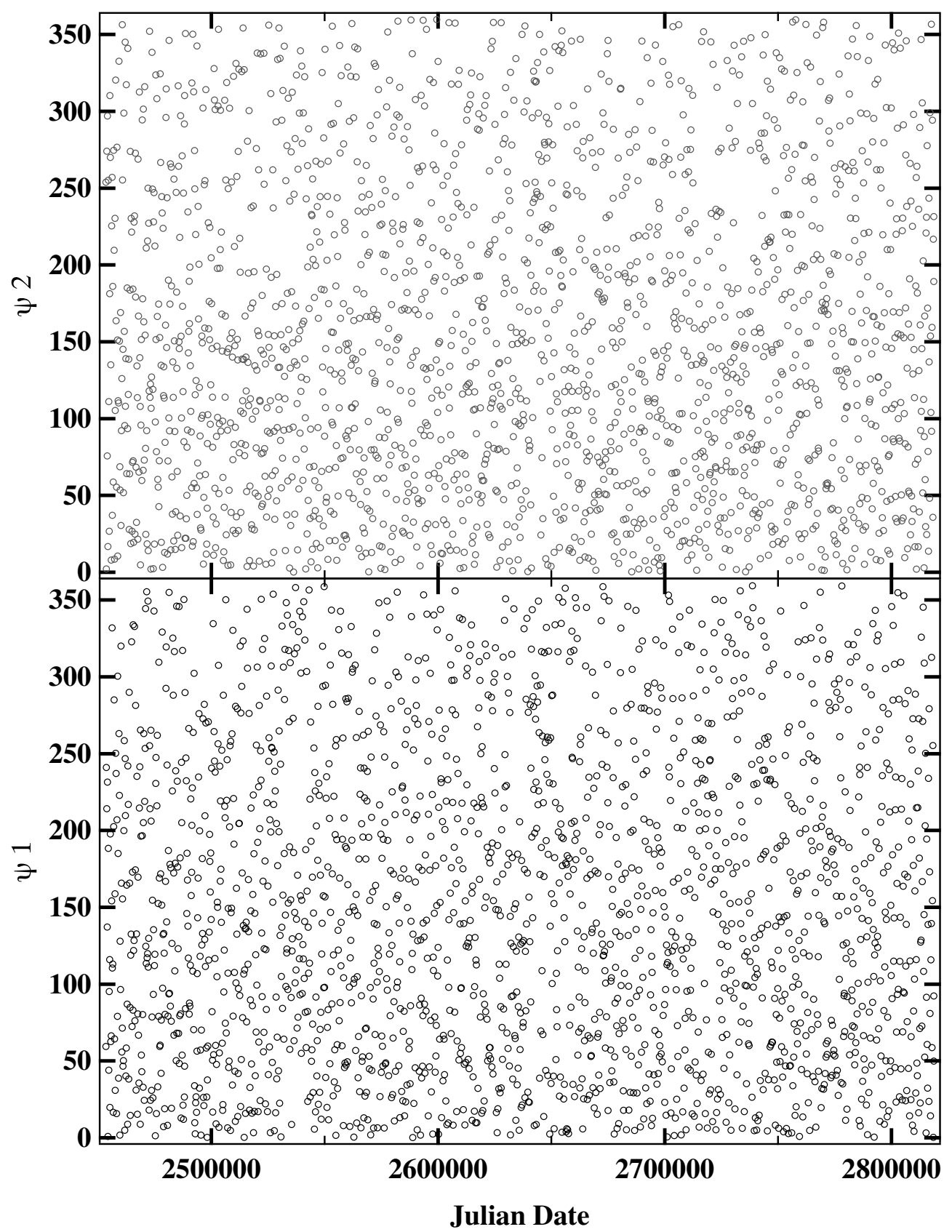

Figure 21. Plot of $\psi$ derived with Equation (10) from the dynamical integration of elements from the solution shown in Table 14 of the HD 128311 system. $\psi$ is circulating, so we do not see the libration that indicates that HD $128311 \mathrm{~b}$ and c are in mean motion resonance.

more as scatter plots, when phased over time (Henry et al. 1995, 2002). Using this equation,

$$
P_{\mathrm{ROT}}=\frac{2 * \pi * R_{\star}}{v \sin i},
$$

and $R_{\star}=$ of $0.76 R_{\odot}$ (from Table 1), and the $v \sin i=$ $3.65 \mathrm{~km} \mathrm{~s}^{-1}$ (from Table 2) we find at an inclination of $90^{\circ}$ a rotation period of $10.53 \pm 1.46$ days, and even shorter if the inclination is less than $90^{\circ}$. If the rotation period is considerably shorter, then we must find an explanation for a rotation period that is shorter than the stable photometric period.

We then turned to our examination of bisectors (Section 4.4). Instead of using individual spectra to calculate bisectors, we computed a CCF which is expected to be more robust. We examined 274 observations to minimize the chance of an anomalous observation dominating the result. Figure 20 shows no correlation between the CCF bisector span and the RV measurements. While it has been shown that there are cases where lack of correlation between bisectors and the RV is not evidence for a planet, Desort et al. (2007) in simulations and Prato et al. (2008) in practice showed that these cases involved stars where the $v \sin i$ is close to or lower than the visible light spectral resolution of the RV observations. In the case of HD 128311, the $v \sin i$ of $3.65 \mathrm{~km} \mathrm{~s}^{-1}$ is close, but larger than $(2 * v \sin i)$ the spectral resolution of the HET/HRS $(R=60,000)$ of around $5 \mathrm{~km} \mathrm{~s}^{-1}$.

While it is tempting to attribute the RV period to the stellar photometric activity or starspots, questions remain as to whether that is a viable explanation. This type of star does not exhibit 
radial pulsations at either 12 days or the shorter $\sim 6$ day period, so we can remove radial pulsations from consideration. The RV orbit determined for HD $128311 \mathrm{~d}$ is eccentric. The photometric variation of HD 128311 is much more stable over a relatively long term of photometric observations that is expected in this type of star. The periods of the RV and photometry are very close but not the same within the errors with large volumes of data analyzed.

One possible explanation for the two observed periods is the presence of differential rotation. We know that HD 128311 is an active, solar-like star so it is reasonable to expect that different latitudes of the star rotate at different periods. Furthermore, if the observed RV and photometric variations are dominated by spots at different stellar latitudes one should see slightly different periods. For instance, if we assume a solar differential rotation law then the spots that dominate the RV and photometric variations would only have to differ in latitude by about $6^{\circ}$.

The lack of correlation between RV and bisector span measurements argues against the spot scenario, but it is not clear if the bisector span measurements are of sufficient quality to be conclusive on this issue. The expressions of Saar \& Donahue (1997) and Hatzes (1996) result in an estimated amplitude for the bisector span variations of $\approx 7 \mathrm{~m} \mathrm{~s}^{-1}$, yet the rms scatter of the bisector span variations is about $20 \mathrm{~m} \mathrm{~s}^{-1}$, or about a factor of three larger. Given the long-lived coherent nature of the photometric variations and its slight difference in period between it and the RV variability, the nature of the 11.2 day RV period is still open. Continued photometric and spectroscopic monitoring of this star is needed to resolve this issue.

It is difficult to explain the long-term stability of the photometry being due to starspots in this type of star in which the rotation period may be shorter. Finally, we do not see correlation between the CCF of the bisectors and the RV, correlation would support starspots or non-radial pulsations. The activity is not likely induced by tidal forces because we would expect the photometric period to be one-half the rotation period. Magnetic interaction producing photometric variations between the possible HD $128311 \mathrm{~d}$ component and the star has not been found in other K star hot Jupiter systems with even shorter periods. If we have indeed found HD $128311 \mathrm{~d}$ and we have an established photometric period that is unusual in nature for this star type, then a mechanism for the relationship between this object and the activity would be interesting and provocative.

\subsection{Are HD 128311 b and $c$ in Resonance?}

The system parameters for HD 128311 derived from the early Lick RV measurements proved to be unstable in dynamical integrations (Vogt et al. 2005). A 2:1 MMR for HD 128311 $\mathrm{b}$ and $\mathrm{c}$ was invoked for stability purposes. Using additional data, Meschiari et al. (2009) derived a Newtonian best fit with the systemic program, but found those orbital parameters were also unstable over a short time period. Further investigations with a bootstrap Monte Carlo procedure of trial fits finally reported a stable fit that was protected by a 2:1 MMR, with a $\theta_{1}$ librating with an amplitude of $\sim 60^{\circ}$ and a circulating $\delta \omega$. However (Voyatzis et al. 2009) using the Butler et al. (2006) orbit, found evidence of libration in all three angles $\theta_{1}, \theta_{2}$, and $\delta \omega$ of $43^{\circ}, 83^{\circ}$, and $103^{\circ}$. Theory suggested that planet-planet scattering was a pathway to the supposed resonance (Barnes \& Greenberg 2006a, 2006b; Raymond et al. 2008). While others produced (Rein \& Papaloizou 2009) a simulation based upon the 2005 Vogt et al. (2005) elements that had stochastic forcing from turbulence and migration but no presence of "rogue" planets.
This suggested that stochastic forces due to turbulence can alter, but not disrupt MMRs. More recently, Lecoanet et al. (2009) reiterated the conclusion of an earlier paper by Adams et al. (2008) that MMRs should be rare, and classified the status of 2:1 MMR of HD 128311 as unresolved.

In contrast to the earlier Keplerian and Newtonian solutions to the available Lick data, with the addition of the large set of HET observations and HST data, and the modeling of an additional signal HD 128311 d, we find a Keplerian solution (see Table 14) that is stable for 10 million years and upon examination, does not show evidence of MMR (see Figure 21), offering support to the conclusions of Adams et al. (2008) and Lecoanet et al. (2009) that MMR's should be rare.

\subsection{Modeling of the Additional Radial Velocity Signal}

Evidence seems to suggest that the third signal, HD 128311 $\mathrm{d}$, found in the RV data set may be a viable planetary candidate. Even if it were some sort of lagging response to the photometric signal, we have modeled it in the RV data, removing its noise from the orbital parameters of HD $128311 \mathrm{~b}$ and c, and produced an orbital architecture that presents contrasting results with previous investigations. Instead of instability, we now have stability for 10 million years, and instead of a theory of MMR in this system, we find no evidence of that in our examination of the $\psi$ 's. Modeling all signals found in our data leads to a very different look at the architecture of this exoplanetary system.

\subsection{The Importance of Astrometric Followup of Extrasolar Systems}

The astrometric determination of the mass of a low-mass companion can decisively characterize it as a planet and reveal its hierarchical position in a planetary system. A good illustration of this fact can be seen from the results of our group for two objects that were previously listed as extra-solar planet candidates: Gliese 876 b and HD 136118 b. Surprisingly each object has been found to belong to a different class: a giant planet and a brown dwarf (Benedict et al. 2002; Martioli et al. 2010). These results demonstrate the importance of the application of complementary techniques in observing extra-solar planetary systems.

\section{CONCLUSIONS}

We have combined 355 new high-cadence RV measurements from the HET, 92 adjusted existing observations from Lick, 112 HST FGS astrometric and photometric observations, and 375 T12 APT photometric observations to clarify the architecture and dynamics of the extrasolar planetary system around the $\mathrm{K}$ dwarf star HD 128311. We have updated a result found in the previous Lick data set for $v$ Andromedae. We offer these final observations.

1. We find an inclination of $55.95 \pm 14.55$ and a true mass of $3.789{ }_{-0.432}^{+0.924} M_{\mathrm{JUP}}$ for HD $128311 \mathrm{c}$ and a lower limit on the inclination of HD $128311 \mathrm{~b}$ of $30^{\circ}$, which could support a coplanar architecture for the system. We have assumed Gaussian errors in the data, and the orbital solution, which is nonlinear, is a local approximation regarding the Gaussian nature of the errors in the parameters we determine.

2. We find an additional RV signal in the data, with a period of $P_{\mathrm{RV}}=11.2210 \pm 0.0008$ days and eccentricity of $0.204 \pm 0.030$, which could be an additional planet d, with a minimum mass of $0.136 \pm 0.007 M_{\mathrm{JUP}}$. Bisector analysis does not confirm stellar activity as the origin of the signal. 
3. We find a consistent long-term photometric signal that is atypical of this type of star that has a period of $P_{\text {phot }}=$ $11.25145 \pm 0.00046$ days, that is very close to, but not within the errors of the RV signal.

4. We find dynamic stability for a span of 10 million years adopting the orbital parameters derived in this paper using Mercury with Bullrisch-Storer integration.

5. We find no evidence of MMR from examination of the $\psi$ 's in this system with the orbital parameters presented in this paper.

6. We find a parallax of $60.532 \pm 0.149$ mas for HD 128311 in good agreement with, but with much lower errors of those found with Hipparcos data.

7. We find that the additional planet found by Curiel et al. (2011) in the previously published data for $v$ Andromedae is a result of $\gamma$ offsets that have since been discovered in the Lick data.

We thank Bill Jefferys for useful discussions about data analysis and statistics. We thank Jacob Bean for the reductions from and implementation of our RV pipeline and Amber Armstrong, Aaron Juarez, and Casey Kyte for their assistance in the processing of this data. We thank our reviewer for helping us bring more clarity to our paper. Support for this work was provided by NASA through grants GO-09971, GO-10103, and GO-11210 from the Space Telescope Science Institute, which is operated by the Association of Universities for Research in Astronomy (AURA), Inc., under NASA contract NAS526555. This publication makes use of data products from the Two Micron All Sky Survey, which is a joint project of the University of Massachusetts and the Infrared Processing and Analysis Center/California Institute of Technology, funded by NASA and the NSF. This research has made use of the SIMBAD database, operated at CDS, Strasbourg, France, and the NASA Astrophysics Data System Abstract Service.

This research is based partially on observations carried out with the Hobby-Eberly Telescope at McDonald Observatory.

Facilities: HET (HRS), HST

\section{REFERENCES}

Adams, F. C., Laughlin, G., \& Bloch, A. M. 2008, ApJ, 683, 1117 Bailer-Jones, C. A. L. 2011, MNRAS, 411, 435

Barnes, R., \& Greenberg, R. 2006a, ApJL, 652, L53

Barnes, R., \& Greenberg, R. 2006b, ApJ, 638, 478

Barnes, S. A. 2007, ApJ, 669, 1167

Bean, J. L., Benedict, G. F., Charbonneau, D., et al. 2008, A\&A, 486, 1039 Bean, J. L., McArthur, B. E., Benedict, G. F., et al. 2007, AJ, 134, 749

Beaugé, C., Michtchenko, T. A., \& Ferraz-Mello, S. 2006, MNRAS, 365, 1160 Beichman, C. A., Bryden, G., Rieke, G. H., et al. 2005, ApJ, 622, 1160 Beichman, C. A., Bryden, G., Stapelfeldt, K. R., et al. 2006, ApJ, 652, 1674 Benedict, G. F., McArthur, B., Nelan, E., et al. 1998, AJ, 116, 429 Benedict, G. F., McArthur, B. E., Feast, M. W., et al. 2007, AJ, 133, 1810 Benedict, G. F., McArthur, B. E., Forveille, T., et al. 2002, ApJL, 581, L115 Benedict, G. F., McArthur, B. E., Gatewood, G., et al. 2006, AJ, 132, 2206 Bessell, M. S., \& Brett, J. M. 1988, PASP, 100, 1134

Black, D. C., \& Scargle, J. D. 1982, ApJ, 263, 854

Bodenheimer, P., Laughlin, G., \& Lin, D. N. C. 2003, ApJ, 592, 555 Bryden, G., Beichman, C. A., Carpenter, J. M., et al. 2009, ApJ, 705, 1226

Butler, R. P., Marcy, G. W., Vogt, S. S., et al. 2003, ApJ, 582, 455

Butler, R. P., Marcy, G. W., Williams, E., et al. 1996, PASP, 108, 500

Butler, R. P., Wright, J. T., Marcy, G. W., et al. 2006, ApJ, 646, 505

Carpenter, J. M. 2001, AJ, 121, 2851

Castelli, F., Gratton, R. G., \& Kurucz, R. L. 1997, A\&A, 318, 841

Chambers, J. E. 1999, MNRAS, 304, 793

Cox, A. N. 2000, Allen's Astrophysical Quantities (4th ed.; New York: AIP)

Crida, A., Sándor, Z., \& Kley, W. 2008, A\&A, 483, 325

Curiel, S., Cantó, J., Georgiev, L., et al. 2011, A\&A, 525, A78
Desort, M., Lagrange, A. M., Galland, F., et al. 2007, A\&A, 473, 983

Donahue, R. A. 1993, PhD thesis, New Mexico State Univ.

Droege, T. F., Richmond, M. W., Sallman, M. P., et al. 2006, PASP, 118, 1666

Eaton, J. A., Henry, G. W., \& Fekel, F. C. 2003, The Future of Small Telescopes in the New Millennium, Vol. 2 (Dordrecht: Kluwer)

Érdi, B., Nagy, I., Sándor, Z., et al. 2007, MNRAS, 381, 33

Figueira, P., Marmier, M., Bonfils, X., et al. 2010, A\&A, 513, L8

Fischer, D. A., Marcy, G. W., Butler, R. P., et al. 2003, ApJ, 586, 1394

Fischer, D. A., \& Valenti, J. 2005, ApJ, 622, 1102

Gabriel, M., \& Grossman, A. S. 1977, A\&A, 54, 283

Gayon, J., \& Bois, E. 2008, A\&A, 482, 665

Gayon-Markt, J., \& Bois, E. 2009, MNRAS, 399, L137

George, S. J., \& Stevens, I. R. 2007, MNRAS, 382, 455

Giuppone, C. A., Beaugé, C., Michtchenko, T. A., et al. 2010, MNRAS, 407, 390

Gonzalez, G., \& Laws, C. 2007, MNRAS, 378, 1141

Gould, A., \& Morgan, C. W. 2003, ApJ, 585, 1056

Goździewski, K., \& Konacki, M. 2006, ApJ, 647, 573

Gray, D. F. 1976, The Observation and Analysis of Stellar Photospheres (Research supported by the National Research Council of Canada; New York: Wiley), 484

Gray, R. O., Corbally, C. J., Garrison, R. F., et al. 2003, AJ, 126, 2048

Hatzes, A. P. 1996, PASP, 108, 839

Hatzes, A. P., \& Cochran, W. D. 1993, ApJ, 413, 339

Hatzes, A. P., \& Cochran, W. D. 1994, ApJ, 432, 763

Hatzes, A. P., Cochran, W. D., \& Johns-Krull, C. M. 1997, ApJ, 478, 374

Heintz, W. D. 1978, Double Stars (Dordrecht: Reidel)

Henry, G. W. 1999, PASP, 111, 845

Henry, G. W., Donahue, R. A., \& Baliunas, S. L. 2002, ApJL, 577, L111

Henry, G. W., Fekel, F. C., \& Hall, D. S. 1995, AJ, 110, 2926

Hernán-Obispo, M., Gálvez-Ortiz, M. C., Anglada-Escudé, G., et al. 2010, A\&A, 512, A45

Huerta, M., Johns-Krull, C. M., Prato, L., et al. 2008, ApJ, 678, 472

Jefferys, W. H. 1990, Biometrika, 77, 597

Jefferys, W. H., \& Barnes, T. G. 1999, in Bayesian Statistics 6, ed. J. Bernardo,

J. Berger, A. Dawid, \& A. Smith (Oxford: Oxford Univ. Press), 777

Jefferys, W. H., Fitzpatrick, M. J., \& McArthur, B. E. 1988, CeMec, 41, 39

Kashyap, V. L., Drake, J. J., \& Saar, S. H. 2008, ApJ, 687, 1339

Koen, C. 2011, MNRAS, 411, 813

Koen, C. 2012, MNRAS, 419, 706

Lawler, S. M., Beichman, C. A., Bryden, G., et al. 2009, ApJ, 705, 89

Lecoanet, D., Adams, F. C., \& Bloch, A. M. 2009, ApJ, 692, 659

Lee, M. H., \& Thommes, E. W. 2009, ApJ, 702, 1662

Mahmud, N. I., Crockett, C. J., Johns-Krull, C. M., et al. 2011, ApJ, 736, 123

Maldonado, J., Martínez-Arnáiz, R. M., Eiroa, C., et al. 2010, A\&A, 521, A12 Mannings, V., \& Barlow, M. J. 1998, ApJ, 497, 330

Martioli, E., McArthur, B. E., Benedict, G. F., et al. 2010, ApJ, 708, 625

McArthur, B., Benedict, G. F., Jefferys, W. H., et al. 1997, in The 1997 HST Calibration Workshop with a New Generation of Instruments, ed. S. Casertano, R. Jedrzejewski, T. Keyes, \& M. Stevens (Baltimore, MD: Space Telescope Science Institute), 472

McArthur, B., Benedict, G. F., Jefferys, W. H., et al. 2002, in The 2002 HST Calibration Workshop : Hubble after the Installation of the ACS and the NICMOS Cooling System, ed. S. Arribas, A. Koekemoer, \& B. Whitmore (Baltimore, MD: Space Telescope Science Institute), 373

McArthur, B. E., Benedict, G. F., Barnes, R., et al. 2010, ApJ, 715, 1203

McArthur, B. E., Benedict, G. F., Jefferys, W. J., et al. 2006, in The 2005 HST Calibration Workshop: Hubble After the Transition to Two-Gyro Mode, ed. A. M. Koekemoer, P. Goudfrooij, \& L. L. Dressel (Greenbelt, MD: Goddard Space Flight Center, NASA), 396

McArthur, B. E., Endl, M., Cochran, W. D., et al. 2004, ApJL, 614, L81

Meschiari, S., Wolf, A. S., Rivera, E., et al. 2009, PASP, 121, 1016

Michtchenko, T. A., Beaugé, C., \& Ferraz-Mello, S. 2008, MNRAS, 387, 747

Moro-Martín, A., Malhotra, R., Bryden, G., et al. 2010, ApJ, 717, 1123

Murtagh, F. 1990, in Errors, Bias and Uncertainties in Astronomy, ed. C. Jaschek \& F. Murtagh (Cambridge: Cambridge Univ. Press), 385

Nelan, E. P. 2007, Fine Guidance Sensor instrument Handbook (16th ed.; Baltimore, MD: STScI)

Paulson, D. B., Saar, S. H., Cochran, W. D., et al. 2004, AJ, 127, 1644

Perryman, M. A. C., Lindegren, L., Kovalevsky, J., et al. 1997, A\&A, 323, L49

Piskunov, N. E., \& Valenti, J. A. 2002, A\&A, 385, 1095

Pourbaix, D., \& Jorissen, A. 2000, A\&AS, 145, 161

Prato, L., Huerta, M., Johns-Krull, C. M., et al. 2008, ApJL, 687, L103

Queloz, D., Henry, G. W., Sivan, J. P., et al. 2001, A\&A, 379, 279

Quillen, A. C. 2006, MNRAS, 365, 1367

Ramírez, I., \& Meléndez, J. 2005, ApJ, 626, 465

Raymond, S. N., Barnes, R., Armitage, P. J., et al. 2008, ApJL, 687, L107 
Reffert, S., \& Quirrenbach, A. 2011, A\&A, 527, A140

Rein, H., \& Papaloizou, J. C. B. 2009, A\&A, 497, 595

Ribas, I., Solano, E., Masana, E., et al. 2003, A\&A, 411, L501

Rocha-Pinto, H. J., \& Maciel, W. J. 1998, MNRAS, 298, 332

Roeser, S., Demleitner, M., \& Schilbach, E. 2010, AJ, 139, 2440

Saar, S. H., \& Donahue, R. A. 1997, ApJ, 485, 319

Saffe, C., \& Gómez, M. 2004, A\&A, 423, 221

Saffe, C., Gómez, M., \& Chavero, C. 2005, A\&A, 443, 609

Saffe, C., Gómez, M., Pintado, O., et al. 2008, A\&A, 490, 297

Sándor, Z., \& Kley, W. 2006, A\&A, 451, L31

Sándor, Z., Kley, W., \& Klagyivik, P. 2007, A\&A, 472, 981

Schlegel, D. J., Finkbeiner, D. P., \& Davis, M. 1998, ApJ, 500, 525

Sozzetti, A. 2005, PASP, 117, 1021

Sozzetti, A., \& Desidera, S. 2010, A\&A, 509, A103

Standish, E. M., Jr. 1990, A\&A, 233, 252

Strassmeier, K., Washuettl, A., Granzer, T., et al. 2000, A\&AS, 142, 275

Takeda, G., Ford, E. B., Sills, A., et al. 2007, ApJS, 168, 297

Tanner, A., Beichman, C., Bryden, G., et al. 2009, ApJ, 704, 109

Trilling, D. E., Bryden, G., Beichman, C. A., et al. 2008, ApJ, 674, 1086
Tull, R. G. 1998, Proc. SPIE, 3355, 387

Tuomi, M., Kotiranta, S., \& Kaasalainen, M. 2009, A\&A, 494, 769

Tuomi, M., Pinfield, D., \& Jones, H. R. A. 2011, A\&A, 532, A116

Valenti, J. A., \& Fischer, D. A. 2005, ApJS, 159, 141

van Altena, W. F., Lee, J. T., \& Hoffleit, E. D. 1995, The General Catalogue of Trigonometric [Stellar] Parallaxes (4th ed.; New Haven, CT: Yale Univ. Observatory) (YPC95)

van Belle Gerard, T., \& von Braun, K. 2009, ApJ, 694, 1085

van Leeuwen, F. 2007, Hipparcos, the New Reduction of the Raw Data (Astrophysics and Space Science Library, Vol. 350; Berlin: Springer)

Vogt, S. S., Butler, R. P., Marcy, G. W., et al. 2005, ApJ, 632, 638

Voyatzis, G., Kotoulas, T., \& Hadjidemetriou, J. D. 2009, MNRAS, 395, 2147

Wittenmyer, R. A., Endl, M., Cochran, W. D., et al. 2009, ApJS, 182, 97

Wright, J. T., Upadhyay, S., Marcy, G. W., et al. 2009, ApJ, 693, 1084

Yong, D., \& Lambert, D. L. 2003, PASP, 115, 796

Zacharias, N., Finch, C., Girard, T., et al. 2009, yCat, 1315, 0

Zacharias, N., Monet, D. G., Levine, S. E., et al. 2004, BAAS, 36, 1418

Zechmeister, M., \& Kürster, M. 2009, A\&A, 496, 577 